\title{
Modelling of Measured Tungsten Spectra from ASDEX Upgrade and Predictions for ITER
}

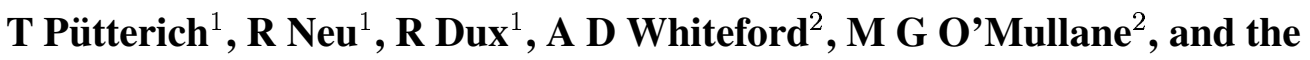 \\ ASDEX Upgrade Team ${ }^{1}$ \\ ${ }^{1}$ Max-Planck-Institut für Plasmaphysik, EURATOM Association, D-85748 Garching, \\ Germany \\ ${ }^{2}$ Department of Physics, University of Strathclyde, Glasgow G4 ONG, UK
}

\begin{abstract}
Tungsten (W) has moved into the focus of fusion research as being a main candidate for the plasma facing components (PFC) of ITER and a future fusion reactor. A main ingredient for understanding the influence of $\mathrm{W}$ as a plasma impurity and its impact on the plasma is the spatially resolved, spectroscopic diagnosis of $\mathrm{W}$. The focus of the experimental investigations at ASDEX Upgrade is on the most intense emissions of W-ions (about I-like $\mathrm{W}^{21+}$ to $\mathrm{Mn}$-like $\mathrm{W}^{49+}$ ) in the VUV to soft X-ray region covering the electon temperature range from about $0.5-5.0 \mathrm{keV}$. The relative shape of the fractional abundances of the ionization stages Se-like $\mathrm{W}^{40+}$ to Ni-like $\mathrm{W}^{46+}$ and of the bundle of ionization stages between Sn-like $\mathrm{W}^{24+}$ to Sr-like $\mathrm{W}^{35+}$ were determined. Calculated fractional abundances using published ionization and recombination rates do not accurately describe the experimental temperature dependence. Adjustments to the recombination rates were calculated to reconcile with the measurements. The spectral features of $\mathrm{W}$ at $0.4-0.8 \mathrm{~nm}$, around $5 \mathrm{~nm}$, between $12-14 \mathrm{~nm}$ and between $10-30 \mathrm{~nm}$ have been recorded and compared to modelling results. The quality of agreement is best for highly charged ionization stages and short wavelengths and decreases for lower charged ionization stages and longer wavelengths. However, in the latter case the predictions manage to reproduce the total emissivity in each considered spectral range and also the rough distribution of emissions versus wavelengths within these spectral ranges. The modelling of the SXR-range at $0.4-0.8 \mathrm{~nm}$ looks very similar to the measurement. Further observations of weaker spectral features between $0.6-0.7 \mathrm{~nm}$, between $1.8-3.5 \mathrm{~nm}$ and at $8 \mathrm{~nm}$ could be attributed to certain ionization stages. The modelling of $\mathrm{W}$-spectra for ITER predicts emissions of Cr-like $\mathrm{W}^{50+}$ to about C-like $\mathrm{W}^{68+}$ at $0.1-0.15 \mathrm{~nm}, 1.8-4.0 \mathrm{~nm}$ and around $8 \mathrm{~nm}$.
\end{abstract}

PACS numbers: $28.52,32.30,34.80,52.20,52.25,52.40,52.55$

Plasma Phys. Control. Fusion

E-mail: Thomas.Puettericheipp.mpg.de

\section{Introduction}

Tungsten is moving back into the focus of spectroscopy for fusion plasmas because it is planned to be used as a plasma facing material in upcoming large tokamaks, such as ITER [1]. As sputtering at the surfaces with plasma contact cannot be avoided completely, tungsten will be an intrinsic impurity in these plasmas. The electron temperatures in ITER will 
span from below $0.1 \mathrm{keV}$ at the edge up to about $30 \mathrm{keV}$ in the core of the plasma. Many different ionization stages - each with a large number of electrons - will contribute to a measured spectrum because spectroscopic measurements are performed on a line of sight (LOS) crossing regions of the plasma with various plasma temperatures. Therefore, the measured spectra often contain many spectral lines from several ionization stages forming spectral features. For interpretation, the identification of the spectral emissions is necessary; the process of understanding the spectra must also be accompanied by model calculations using atomic data. This analysis supports the disentangling of the experimental spectra and yields a quantitative understanding of the measurement. However, atomic data for high$\mathrm{Z}$ elements are scarce and elaborate to produce, because of the high computational effort needed to perform calculations for these ions as well as more pronounced relativistic effects. Theoretical data for the ionization equilibrium and the excitation rate coefficients along with the energy level structure and transition probabilities are the basic input to model the emissions of tungsten. Neighbouring ionization stages with similar electron structure emit spectral lines at similar wavelengths. This leads to the spectral features mentioned above, especially, when open d- or f-orbitals are involved. First observations of this phenomenon have been performed in the ORMAK [2] and PLT [3] tokamaks in the 1970s, where the quasicontinuum emission around $5 \mathrm{~nm}$ was reported. Further investigations have been performed at the ASDEX Upgrade tokamak [4, 5, 6, 7, 8], which also revealed spectral emissions at electron temperatures above $2 \mathrm{keV}$ in the soft X-ray and the VUV. The ionization equilibrium has been analysed in [4] by using the ADPAK ionization and recombination data from the average ion model $[9,10]$. Analysis of the VUV spectrum in EBIT devices were conducted at the Berlin [11], the Lawrence Livermore National Laboratory [12] and National Institute of Standards and Technology [13] EBITs. These and supplementary investigations at ASDEX Upgrade [14] identified the emitting ionization stages of most spectral lines between 4 and $15 \mathrm{~nm}$. This allowed for the analyses presented below which determine the abundance of ionization stages versus electron temperatures $(1-4.5 \mathrm{keV})$ for fusion-relevant electron densities. Furthermore, several new spectral features emitted by tungsten are measured at ASDEX Upgrade and investigated by comparison to modelling results. The model is based on atomic data produced by the Cowan code [15] using a plane-wave Born approximation for electron impact excitation. On the experimental side, ASDEX Upgrade is offering tungsten spectra emitted from various plasma discharges, as all plasma facing components (PFCs) have been stepwisely covered with tungsten [16] (starting in 1999 and reaching $100 \%$ in 2007). Details of the investigations can be found in [7], however, the calculations used in [7] have been improved and limitations of the code calculations have been overcome, such that for a single ionization stage up to a million transitions can be included. Especially, for the ionization stages with open $\mathrm{d}$ - and f-shells this led to an improvement of the modelling results. The paper aims to improve the knowledge about the spectra of tungsten ions, such that features of these spectra can be used for quantitative measurements in a fusion plasma. This effort includes measurements of the ionization equilibrium and the measurement and modelling of spectra to benchmark the quality of atomic data and to increase the understanding of the $\mathrm{W}$ spectra. In the next section, the investigations concerning the ionization balance are presented. 
In section 3, the modelling of spectra is described, while in section 4 the measurements of the brightest spectral features are presented and modelled. In section 5, observations of additional spectral features are presented. Before the summary is given in section 8 , an outlook to future tungsten spectra for JET and ITER is given (section 6) and recommendations on diagnostic lines for fusion plasmas in general are given (section 7).

\section{The Ionization Equilibrium of Tungsten}

For the interpretation of spectral emissions and their modelling, it is necessary to know the abundance of each tungsten ionization stage along the line of sight (LOS) of the measurement. In a fusion plasma, the fractional abundance is determined by the recombination and ionization rates along with the plasma transport. It was found earlier [4] that within a certain plasma radius $\left(\rho_{p o l} \leq 0.7\right)$ plasma transport affects the ionization equilibrium of $\mathrm{W}$ only weakly. At these radii, the typical transport times are large in comparison to the equilibration time of the ionization balance. Outside this radius, fast transport events and an increased diffusive transport are observed, which make the interpretation of impurity radiation more challenging. Below, the focus is placed on the central part of the plasma. The ionization equilibrium is calculated by the transport code STRAHL $[17,18]$ in which typical transport coefficients are used (e.g. $[19,20]$ ). Due to the fact, that ionization and recombination rates for high-Z elements are large compared to transport times, transport plays only a minor role for the ionization equilibrium (within $\rho_{\text {pol }} \leq 0.7$ ). Therefore, no detailed explanation about transport coefficients is provided in the following. Different sets of ionization and recombination rates are discussed and compared to measurements. A large experimental dataset is available from ASDEX Upgrade for various types of plasma discharges. In particular discharges with impurity accumulation are very useful to determine the abundance range of an ionization stage as explained in the following paragraphs. During impurity accumulation, a very small central part of the plasma exhibits tungsten concentrations which are higher than in the rest of the plasma by factors of up to 100 [21, 22]. Line-integrated measurements by bolometers and spectrometers are dominated from the plasma region where accumulation takes place (see figure 1). The electron temperature in this small region as measured by electron cyclotron emissions and Thomson scattering, is spatially about constant in the accumulation volume because the spatial extension of the region is small with respect to gradients in electron temperature. Moreover, the radiative losses lead to a flattening of the electron temperature profile. This allows for taking quasi-local spectra which are associated with a single electron temperature. Conclusions about the fractional abundance of ionization stages can be drawn, because the following two issues are well understood:

Firstly, the intensity of spectral lines emitted by highly-ionized tungsten (i.e. in this work ionization stages above $\sim \mathrm{W}^{20+}$ ) depends strongly on the fractional abundance of the emitting ionization stage, while additional factors are relatively small and can be corrected or neglected at all. For illuminating the latter statement, the emissivity $\epsilon_{\text {line }}$ of a measured spectral line is considered in the following, using $n_{e}$ as local electron density, $n_{W}$ as local tungsten density, $f_{Z}$ as the local fractional abundance of the emitting ionization stage $\mathrm{Z}$ and $P E C_{\text {line }}$ as the 


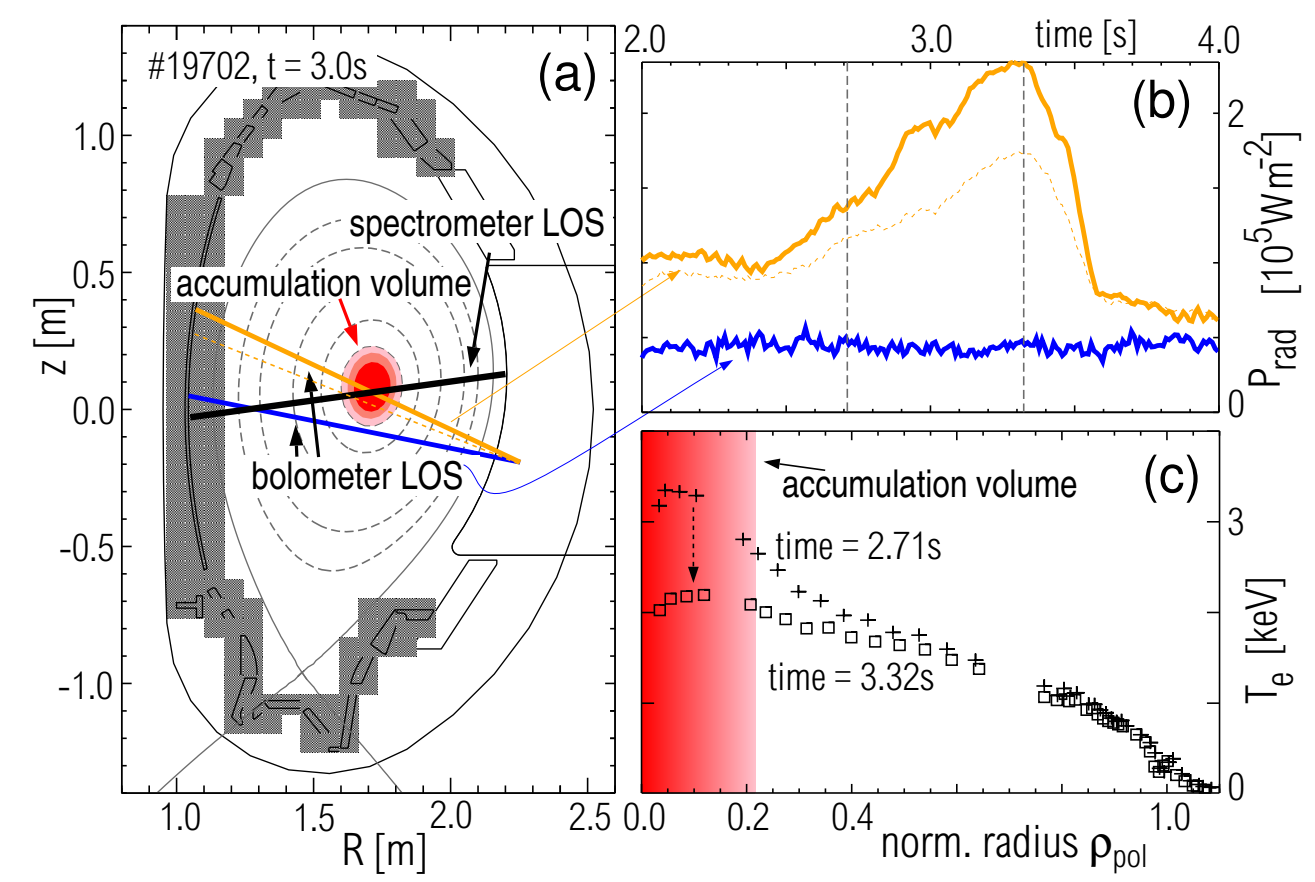

Figure 1. (a) Setup for the measurements. A magnetic equilibrium with three lines of sight (LOS) of the bolometer system and the LOS of the spectrometer are presented. On flux surfaces the plasma parameters are spatially constant. (b) Time traces for the lineintegrated measurements (corresponding to the presented LOS of the bolometer) of total radiated power diagnosing impurity accumulation; (c) Electron temperature profiles for two timepoints (indicated in part (b)) measured during impurity accumulation using the electron cyclotron emission.

photon emissivity coefficient of the measured spectral line.

$$
\epsilon_{\text {line }}=n_{e} n_{W} f_{Z} P E C_{\text {line }}\left(n_{e}, T_{e}\right)
$$

The total radiated power density $\epsilon_{\text {tot }}$ is subject to bolometer measurements and can be written as

$$
\epsilon_{t o t}=n_{e} n_{W} L_{W}\left(n_{e}, T_{e}\right)
$$

using $L_{W}$ as the cooling factor of tungsten. Thus:

$$
f_{Z}=\frac{\epsilon_{\text {line }}}{n_{e} n_{W} P E C_{\text {line }}\left(n_{e}, T_{e}\right)}=\frac{\epsilon_{\text {line }}}{\epsilon_{\text {tot }}} \frac{L_{W}\left(n_{e}, T_{e}\right)}{P E C_{\text {line }}\left(n_{e}, T_{e}\right)} .
$$

The density dependence of $L_{W}\left(n_{e}, T_{e}\right)$ and $P E C_{\text {line }}\left(n_{e}, T_{e}\right)$ for the actual application is small and can be neglected. As pointed out in [14], the relative population of the levels, which decay by emitting the photons, is nearly independent inside the electron temperature range in which the spezific ionization stage is abundant. In principle, exceptions to this general rule exist, but in the present section, spectral lines have been used for which it applies. Therefore, the temperature dependence of $P E C_{\text {line }}\left(n_{e}, T_{e}\right)$ can also be neglected, while that of $L_{W}\left(n_{e}, T_{e}\right)$ must be taken into account, using the caluclated $L_{W}[9,10,23]$. The latter corrections are smaller than a factor of 3 . 
Secondly, the ionization equilibrium inside the accumulation region is very well described without taking transport into account, such that the difference is experimentally not distinguishable. This fact was generally described above, but is treated here specifically for impurity accumulation in more detail. In figure 2, the fractional abundances inside the accumulation volume for an electron temperature of $4.1 \mathrm{keV}$ (case is taken from experiment) are presented for different transport coefficients and the ionization/recombination rates, which are later identified to describe the experimental data best. The accumulation is assumed to be the result of the neoclassical inward drift. The effects of the latter on the $\mathrm{W}$ profile depends on the diffusion coefficient which may be a mixture of neoclassical and anomalous contributions. The neoclassical inward drift has been evaluated from the measured plasma parameters using NEOART [24]. NEOART calculates the collisional transport coefficients for an arbitrary number of impurities including collisions between all components. The code solves the set of linear coupled equations for the parallel 'velocities' in arbitrary toroidally symmetric geometry for all collision regimes. The classical fluxes are given by Eqs.(5.9) and (5.10) in [25]. The equations for the banana plateau contribution are equal to that used in [26]. The Pfirsch-Schlüter contribution is calculated from the coupled equations (6.1-2) and (6.14-15) in [25], as described in [27]. The anomalous contributions to the diffusion coefficients have been chosen such that the impurity density profile in the transport model matches the experimentally measured one (central diffusion coefficient: $0.1 \mathrm{~m}^{2} / \mathrm{s}$ ). This choice also allows to reproduce the time scale of impurity transport towards the accumulation region within a factor of 2 . For these transport coefficients, the ion abundances indicated in figure 2 with the solid line have been evaluated. They do not exhibit any visible difference to the transport-free abundances denoted by the black diamonds. When transport coefficients clearly larger than the neoclassical ones are used and the anomalous diffusion coefficient is increased proportionally in the transport model (3 times larger and 10 times larger transport coefficients (dotted, blue curve and dashed, orange curve)) still no differences are visible for the most abundant ionization stages. Differences for the enhanced transport scenarios occur only for the ionization stages for which the accumulation region is too hot, but for which the increased inward transport leads to an increase of their abundance. However, the effect is only visible, if the amount of the ionization stage, which is abundant in the central plasma is small (about factor 100 below the most abundant stage). Therefore, the measurements in the accumulation region allow to investigate the transport-free situation, in which only the ionization and recombination rates determine the fractional abundance of each ionization stage.

In figure 1(a) the geometrical setup of measurements is presented. The impurity accumulation is diagnosed by bolometer measurements of which three LOS are depicted in the figure. One (thick, solid, orange) of them is observing the central part of the plasma and an other (thick, solid, blue) is slightly off-central. The difference between the measured signal of the two LOS at each timepoint is associated to a localized peak of the radiated power density in the central part of the plasma and is suited to quantify the impurity accumulation. Due to the fact that the plasma parameters are spatially constant along the magnetic flux surfaces, already a measurement on a few LOS covering the central plasma region is sufficient to 


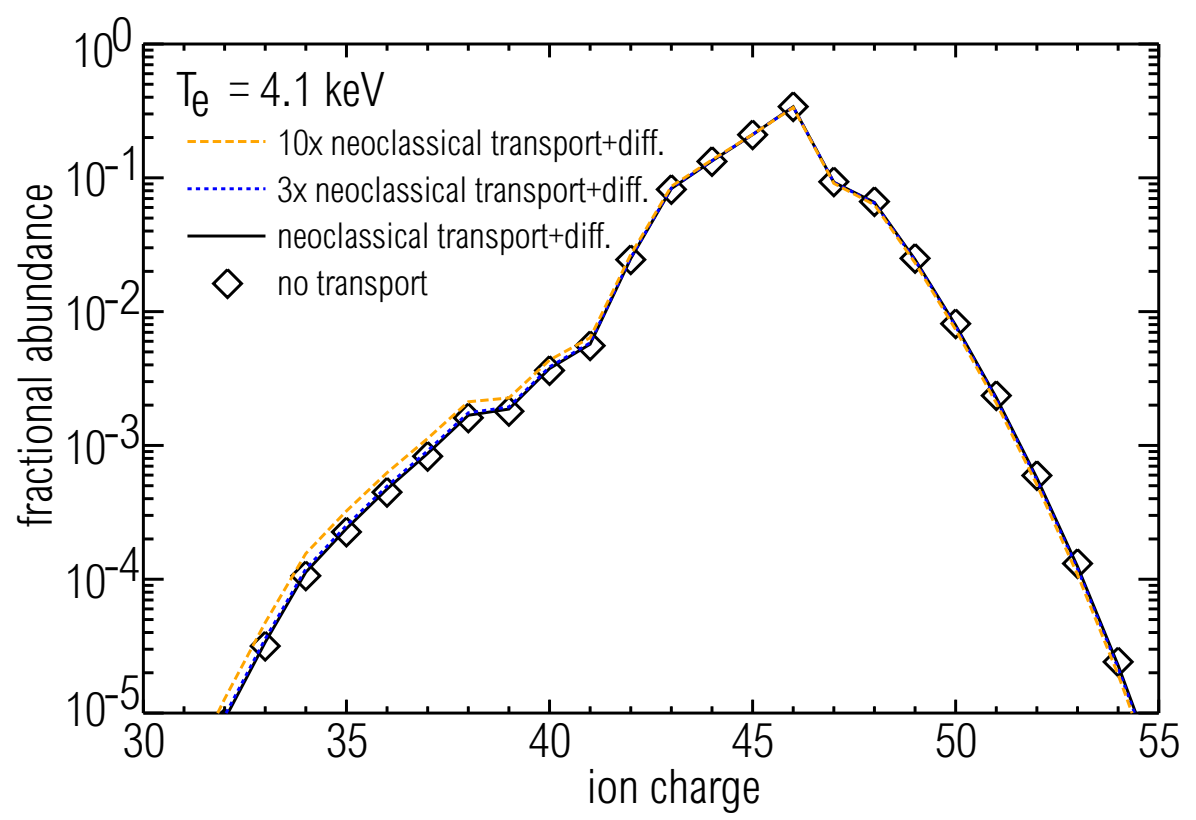

Figure 2. Effects of transport on the ion abundances within the accumulation region. Neoclassical inward drifts are evaluated for tungsten using the measured electron profiles. Diffusion due to turbulence is adjusted such that magnitude and time scale of impurity accumulation match the experiment within a factor of 2 . A minor effect on the equilibrium is only visible for transport coefficients much larger than predicted for a neoclassical inward drift.

quantify the magnitude and radial extent of the radiation peaking at each point in time. It may be noted that earlier investigations [19] revealed poloidal asymmetries (deviations of the radiation profile from the magnetic symmetry) for high- $Z$ elements due to centrifugal effects [28]. Such effects only weakly influence the measurements presented here, because all the used LOS are directed almost radially integrating over such asymmetries. Another concern might be the accuracy of the alignment of bolometer LOS compared to the spectrometer LOS. The uncertainty of the magnetic flux surfaces at the plasma center exhibits uncertainties below $2 \mathrm{~cm}$ as known from comparison to measurements from soft X-ray cameras. The thin, dashed, orange LOS in figure 1(a) depicts a bolometer LOS, which crosses the plasma center $4 \mathrm{~cm}$ below the bolometer LOS which is equivalent to the spectrometer LOS (thick, solid, orange). The corresponding measurement is depicted in figure 1(b) showing that even for an exaggerated spatial deviation of $4 \mathrm{~cm}$ the latter result for the realitve shape of $f(Z)$ is influenced by less than a relative correction of about $30 \%$ (keeping in mind, that the quantity, which is entering the results of $f(Z)$ is the relative behaviour of the radiated power measured on the central LOS (thick, solid, orange) minus the the radiated power on the off-central LOS (thick, solid, blue), see below). The central part of the plasma is also monitored by several spectrometers (e.g. VUV SPRED spectrometer [29], grazing incidence spectrometer [30], scanning Bragg crystal spectrometer [31] and a highly resolving Johann spectrometer [8]). The LOS of a grazing incidence spectromter is depicted in figure 1(a). The spectral lines from the accumulation region dominate the spectrum, because the corresponding lines 

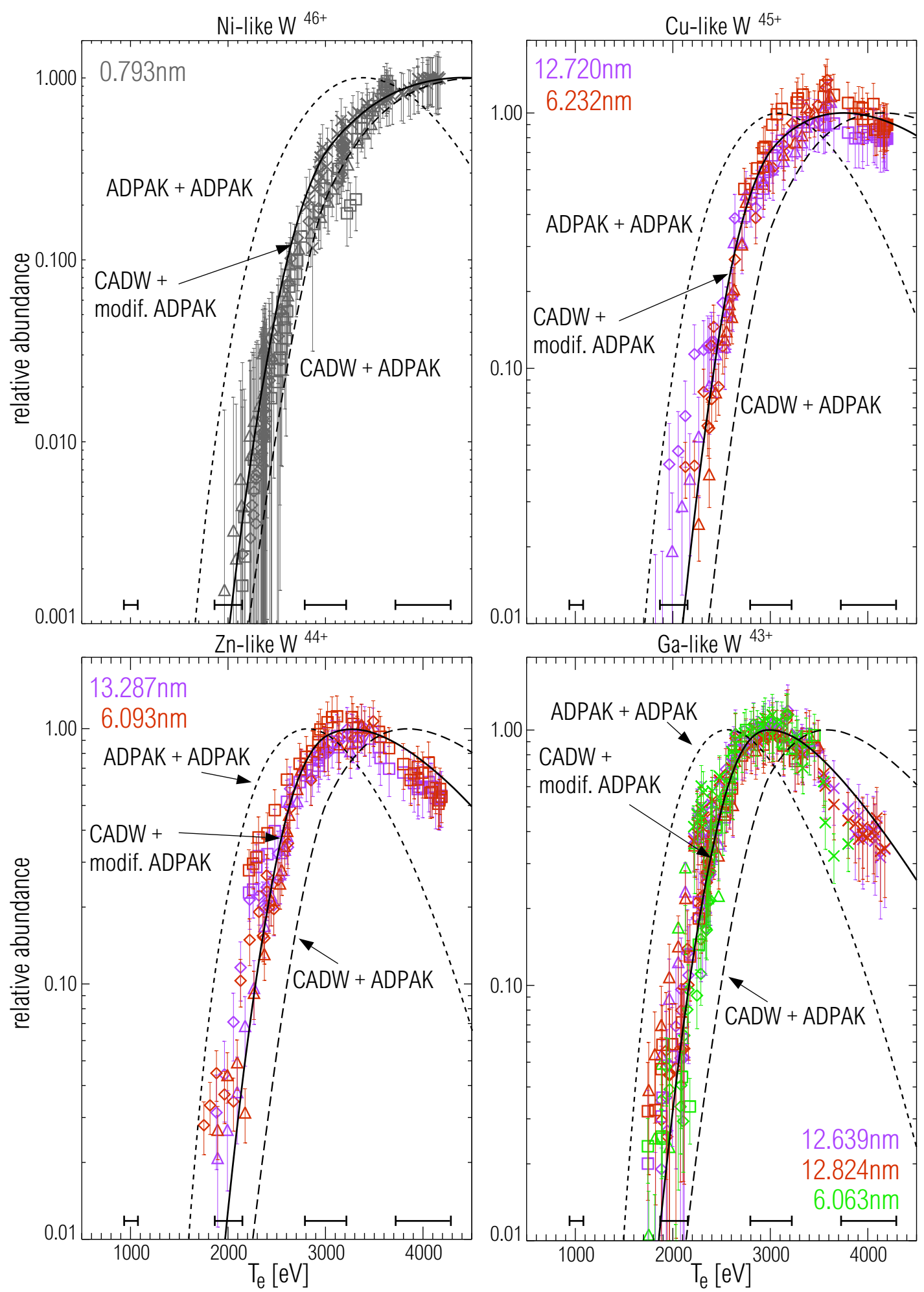

Figure 3. Relative abundances of Ni-like $\mathrm{W}^{46+}$ to Ga-like $\mathrm{W}^{43+}$ derived from theoretical data (lines) and from measurement (symbols). The measurements originate from impurity accumulation phases. Further information in the text. 

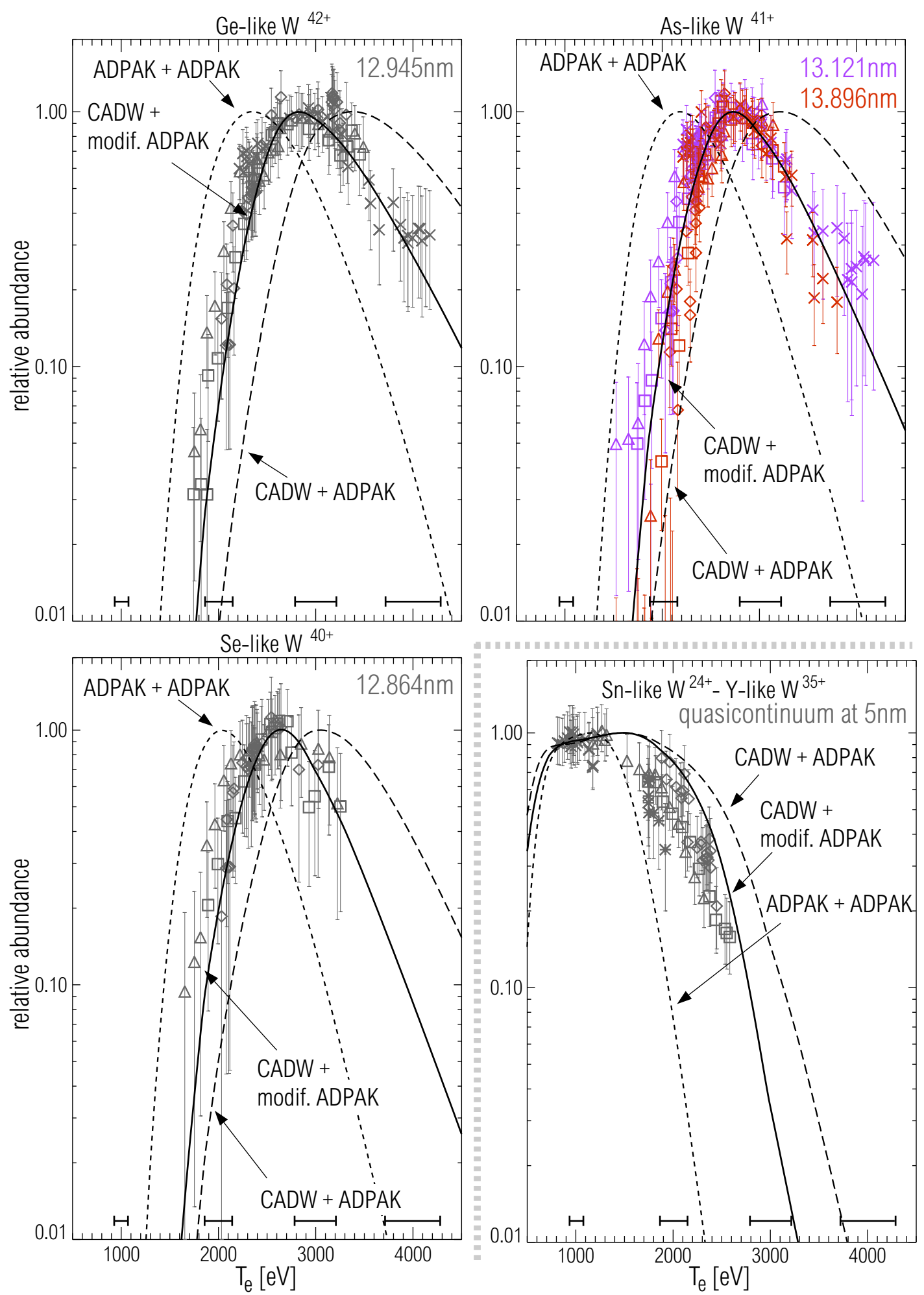

Figure 4. Relative abundances of Ge-like $\mathrm{W}^{42+}$ to Se-like $\mathrm{W}^{40+}$ and the relative behaviour of the compound emissions around $5 \mathrm{~nm}$ of $\mathrm{Y}$-like $\mathrm{W}^{35+}$ to Sn-like $\mathrm{W}^{24+}$ derived from theoretical data (lines) and from measurement (symbols). The measurements originate from impurity accumulation phases. Further information in the text. 
emitted outside the plasma center are emitted at regions with $a \approx 20-100$ times lower tungsten concentration. For several phases, the intensities of spectral lines (corresponding to $\left.\int_{L O S} \epsilon_{l i n e}\right)$ have been recorded as a function of time, while the bolometer was used to determine $\int_{L O S} \epsilon_{t o t}$ on an equivalent LOS. The absolute values of the integrals and the absolute values of $\frac{L_{W}}{P E C_{\text {line }}}$ are not considered here, as the uncertainties in the absolute values might be considerable. However, the relative behaviour of $\int_{L O S} \epsilon_{l i n e} / \int_{L O S} \epsilon_{t o t}$ using corrections for the temperature dependence of $L_{W}$ gives the relative behaviour of $f_{Z}$ in the accumulation region as the integrands of both integrals are dominated by emissions from that volume. As pointed out above the corrections due to the temperature dependence of $L_{W}$ are smaller than factor of 3 owing to a change of the ionization equilibrium and connected to that a change of the radiated power by spectral lines and radiative recombination. The factor of 3 corresponds to the change of $L_{W}$ between electron temperatures of $1 \mathrm{keV}$ to $5 \mathrm{keV}$. The error bars for the relative shape of $f_{Z}$ are determined partly by the spectroscopic uncertainties and partly by the estimated uncertainies in determining the correct time behaviour of $\int_{L O S} \epsilon_{t o t}$ and $L_{W}$. For the latter $20 \%$ of the central radiation are assumed. At the same time the electron temperature in the accumulation region is recorded, which allows for monitoring the ionization equilibrium at different electron temperatures. For the whole analysis discharges were chosen which exhibit a continuous behaviour of accumulation and drop in electron temperature, i.e. no sawteeth are observed. For two time points (see vertical lines in figure 1(b)), the electron temperature profiles are plotted in figure 1(c) as measured by electron cyclotron radiation diagnostics. In figure 3 and 4 , the measured $f_{Z}$ are presented for several ionization stages, while all data points have been normalized (for each spectral line a single factor, for all discharge phases), such that the maximum of the measured curve reaches 1 . Within the diagramm of each ionization stage the same symbols correspond to the same occurence of impurity accumulation, while the colors indicate the spectral line or feature which was interpreted. For the whole analysis, the electron temperature measurement was supplied by the electron cyclotron radiation diagnostics, because it supplies high quality profile measurements. The diagnostics is calibrated each year correcting changes of each channel individually. The changes of calibration factors between two calibrations plus the statistical uncertainties are smaller than $7 \%$ (indicated at the bottom of each diagramm of figures 3 and 4), which is also supported by comparisons to the Thomson scattering diagnostics. For better comparison to the experimental data, the theoretical data derived from three combinations (described below) of ionization and recombination rates have been also normalized to 1 for better comparison to the experimental data. A special case is the fractional abundance derived from the emissivity of the quasicontinuous emission around $5 \mathrm{~nm}$ which is emitted by ionization stages around Sn-like $\mathrm{W}^{24+}$ to $\mathrm{Y}$-like $\mathrm{W}^{35+}$. Here, all contributing ionization stages have been added and a weighting according to the predicted brightnesses of the spectral lines (from calculations of atomic data - see next section) of each ionization stage was applied. The experimental data shows the integrated intensity of the quasicontinum at $5 \mathrm{~nm}$. For the other ionization stages, the following spectral lines have been used (if not specified, uncertainty of the wavelength is $0.005 \mathrm{~nm}): \mathrm{W}^{40+}$ : $12.864 \mathrm{~nm} ; \mathrm{W}^{41+}$ : $13.121 \mathrm{~nm}, 13.896 \mathrm{~nm} ; \mathrm{W}^{42+}: 12.945 \mathrm{~nm} ; \mathrm{W}^{43+}$ : $6.063 \mathrm{~nm}, 12.639 \mathrm{~nm}, 12.824 \mathrm{~nm}$; $\mathrm{W}^{44+}: 6.093 \mathrm{~nm}, 13.287 \mathrm{~nm} ; \mathrm{W}^{45+}: 6.232 \mathrm{~nm}, 12.720 \mathrm{~nm}$; 
$\mathrm{W}^{46+}: 0.793 \pm 0.001 \mathrm{~nm}$

For comparison with the experimental data, three combinations of ionization and recombination rates are used. The first makes use of improved [4] ADPAK rates [9] for ionization and the original ADPAK rates for recombination. In the following figures 'ADPAK+ADPAK' is used for labelling the fractional abundances which result from that combination. A second, more elaborate option is available for the ionization rates, which originate from configuration-averaged distorted-wave calculations [32] (CADW). The calculations using them in combination with the recombination data from ADPAK yields the fractional abundances labelled with 'CADW+ADPAK'. A third data set (labelled 'CADW+modif. ADPAK') is derived from the latter combination, while the recombination rates have been scaled by temperature independent factors, such that the best agreement with the experimental data (i.e. the relative abundance versus electron temperature, but also the ion abundance with respect to neighbouring ionization stages as investigated below) is achieved. The factors are given in table 1 . The recombination rates of more than the observed ionization stages have been changed, because also the abundance of lower and higher charged ionization stages influence the abundance of the observed ionization stages. The adjustments are done

\begin{tabular}{|c|c|c|c|c|c|}
\hline Recombined Ion & $\mathrm{F}_{\text {corr }}$ & Recombined Ion & $\mathrm{F}_{\text {corr }}$ & Recombined Ion & $\mathrm{F}_{\text {corr }}$ \\
\hline Xe-like W ${ }^{20+}$ & 0.97 & Mo-like $\mathrm{W}^{32+}$ & 1.62 & Zn-like $\mathrm{W}^{44+}$ & 0.47 \\
\hline I-like $\mathrm{W}^{21+}$ & 1.07 & Nb-like W ${ }^{33+}$ & 1.62 & Cu-like $\mathrm{W}^{45+}$ & 0.39 \\
\hline Te-like $\mathrm{W}^{22+}$ & 1.17 & Zr-like $\mathrm{W}^{34+}$ & 2.25 & Ni-like $\mathrm{W}^{46+}$ & 1.78 \\
\hline Sb-like W ${ }^{23+}$ & 1.27 & Y-like $\mathrm{W}^{35+}$ & 2.15 & Co-like W ${ }^{47+}$ & 0.60 \\
\hline Sn-like $\mathrm{W}^{24+}$ & 1.37 & Sr-like $\mathrm{W}^{36+}$ & 2.05 & Fe-like $\mathrm{W}^{48+}$ & 0.99 \\
\hline In-like $W^{25+}$ & 1.47 & Rb-like W $^{37+}$ & 1.76 & Mn-like $\mathrm{W}^{49+}$ & 0.99 \\
\hline Cd-like $\mathrm{W}^{26+}$ & 1.58 & Kr-like $\mathrm{W}^{38+}$ & 1.76 & Cr-like $\mathrm{W}^{50+}$ & 0.96 \\
\hline Ag-like $\mathrm{W}^{27+}$ & 1.52 & Br-like W $^{39+}$ & 1.10 & V-like $\mathrm{W}^{51+}$ & 0.95 \\
\hline Pd-like W ${ }^{28+}$ & 1.52 & Se-like $\mathrm{W}^{40+}$ & 1.33 & Ti-like $\mathrm{W}^{52+}$ & 0.94 \\
\hline Rh-like W ${ }^{29+}$ & 1.62 & As-like $\mathrm{W}^{41+}$ & 0.34 & Sc-like $\mathrm{W}^{53+}$ & 0.95 \\
\hline Ru-like $\mathrm{W}^{30+}$ & 1.62 & Ge-like $\mathrm{W}^{42+}$ & 0.26 & Ca-like $\mathrm{W}^{54+}$ & 0.97 \\
\hline Tc-like $\mathrm{W}^{31+}$ & 1.62 & Ga-like $\mathrm{W}^{43+}$ & 0.45 & K-like $\mathrm{W}^{55+}$ & 0.98 \\
\hline
\end{tabular}

Table 1. Correction factor $\mathrm{F}_{\text {corr }}$ of the ADPAK recombination rate coefficients which are introduced for improved agreement with the experimental findings (temperature dependence of fractional abundances, relative weight of ionization stages).

to give an indication where the discrepancies are largest and to provide a set of rates, which is in agreement with experiment within the uncertainties. The measurements, however, are only available for most of the ionization stages between Sn-like $\mathrm{W}^{24+}$ and Ni-like $\mathrm{W}^{46+}$. Due to this fact and since the uncertainties of this measurements leave some flexibility, the adjustments are not a unique solution and have been performed by hand. In principle, differences could also originate from recombination rates, which have a different shape versus electron temperature or due to different ionization rates - as the described discrepancies are not understood the choice of correction has no preference. Nevertheless, the investigations provide valuable feedback to fundamental cross section calculations.

Apart from the electron temperature dependence of the fractional abundances of 
ionization stages, also the absolute values could be investigated by comparing the total brightness of an ensemble of spectral lines of one ionization stage to that of other ionization stages using predictions for their brightness from atomic data. An ensemble of lines is chosen to rely less on the accuracy of the calculated emissivity for a single line. For these investigation, plasma discharges are used which exhibit a flat radial tungsten profile, as can be judged from the radiation profile and a tungsten diagnostics based on calibration discharges - described in [4]. In figures 8, 9 and 11 (discussed in section 4), the used spectral lines for each ionization stage are highlighted by small, black arrows. Some spectral lines have been omitted for this comparison if the identification was unclear or line blending occured. The details of the atomic data calculations for predicting line intensities will be presented in the next section, however, the impact on the investigations on the ionization stage abundances is discussed here. In detail, the emissions from each ionization stage have been summed up for the soft X-ray region and two VUV regions. For each spectral region, this sum is then divided by the predicted line intensities resulting from a detailed modelling. All ratios are normalized such that the ratio for $\mathrm{Zn}$-like $\mathrm{W}^{44}$ equals 1 . The latter normalization is because the absolute tungsten concentration is a free parameter when looking at the fractional abundances of each ionization stage. In figure 5, the results are presented for the data set 'CADW+ADPAK'
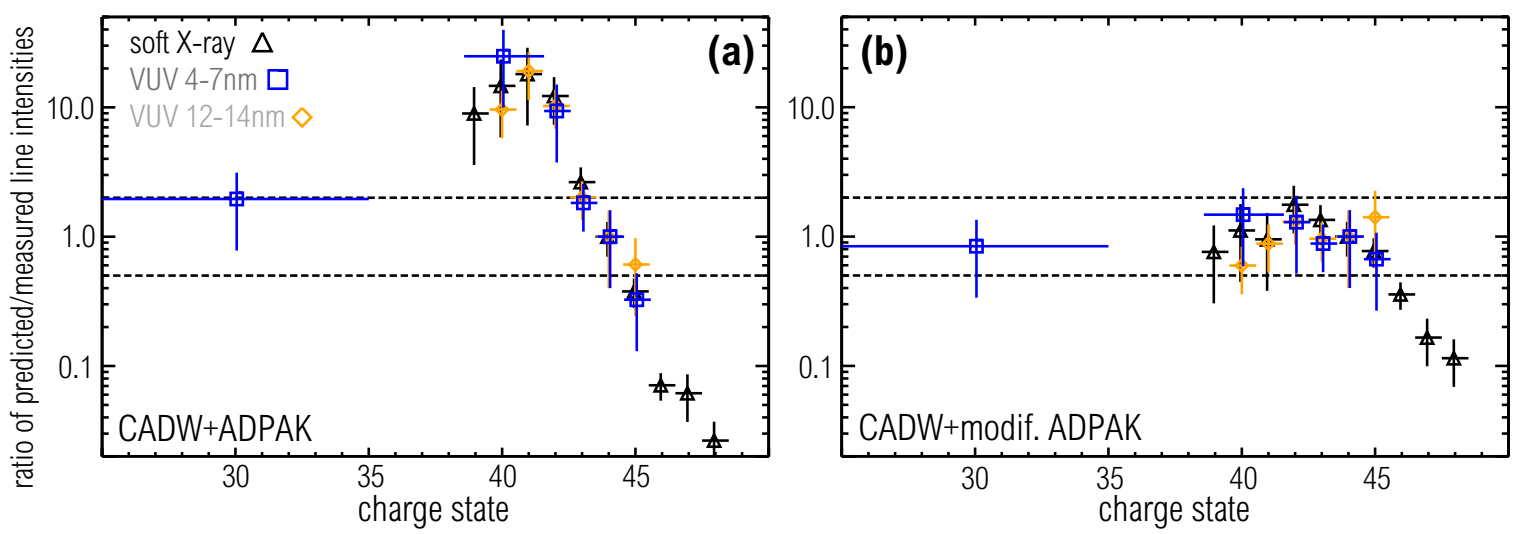

Figure 5. (a) Ratios of predicted to measured line intensities for different wavelength ranges versus ionization stage using the 'CADW+ADPAK' ion balance (see figure 6). Several lines of an ionization stage are summed in the model and in the spectrum to reduce the uncertainties that could occur for a single spectral line. (b) Similar data as (a), but using the data set 'CADW+modif. ADPAK' which is described in the text. Dashed lines correspond to 'factor of 2'-margin around 1 introduced to guide the eye.

and the set 'CADW+modif. ADPAK' demonstrating that the adjustments applied to the ADPAK recombination rates lead to an improvement also for the absolute values of fractional abundances. Whereas for the 'ADPAK+CADW' case, deviations for the ionization stages below $\mathrm{Cu}$-like $\mathrm{W}^{45+}$ (including $\mathrm{W}^{45+}$ ) of up to a factor of 30 are observed, a reduction of that deviation to less than a factor of 2 is achieved with the modified recombination rates. For the ionization states above Ni-like $\mathrm{W}^{46+}$ (including $\mathrm{W}^{46+}$ ) the model underestimates the emissions for the unmodified case by up to a factor of 50 and the modifications reduce this maximum discrepancy to a factor of 10 . The discrepancy is smallest for Ni-like $\mathrm{W}^{46+}$ (about 
factor of 3) and inreases for higher charge. These discrepancies could not be corrected by the modification of the recombination rates using energy independent factors without sacrifying the above presented agreement in the relative shape of the abundance shells versus electron temperature. Possibly, energy dependent corrections are necessary. It should be noted, that weak impurity accumulation with an impurity peaking factor of about 3 might be undetected and cannot be excluded. This would lead to an increased contribution from exactly the ionization stages above $\mathrm{Ni}$-like $\mathrm{W}^{46+}$.

In figure 6, a comparison of the resulting ionization equilibria is presented. The two ionization equilibria depicted in figure 6 (b) and (c) exhibit a less continuous behaviour than that of the 'ADPAK+ADPAK' data in part (a). This structure corresponds to the more detailed information entering into the ionization rates, while additional structure is added by the adjustments of recombination rates. It may be noted that the combination of absolute and relative information on the fractional abundances also restricts the fractional abundances of ionization stages, for which no direct measurement is available, like Sr-like $\mathrm{W}^{36+}$ to $\mathrm{Kr}$ like $\mathrm{W}^{38+}$. Since the abundances of neighbouring ionization stages is measured to be high, the abundance of these ionization stages is suppressed. This consequence is in agreement with not observing spectral lines emitted by these ionization stages in the VUV region where bright enough lines are predicted by the code calculations. This also gives further support to the modifcations to the ADPAK recombination rates, as the unmodified version would predict clearly visible spectral lines in the modelled spectra emitted by these ionization stages. In figure 7 , some of the chosen rates are presented.

\section{The modelling of spectra}

The modelling of the spectra involves several steps. With the Cowan code [15] fundamental properties of the emitting ions have been evaluated. For the ions of interest, energy levels and transition probabilities were calculated. Additionally, cross sections for electronimpact excitation have been targeted with the Cowan code by using the plane wave Born approximation resolving all levels of the target ions. All the data were then fed into a collionalradiative model to derive emissivities of spectral lines in a plasma for densities of $10^{18} \mathrm{~m}^{-3}-$ $10^{20} \mathrm{~m}^{-3}$. All codes are part of or have been integrated into a program suite provided by the ADAS (Atomic Data and Analysis Structure) Project [33]. Therefore, data originating from the present work will be labelled with 'ADAS'. For comparison to measurements, these data were then used to produce synthetic spectra in the real geometry. The integration along the LOS of the spectrometer was numerically performed taking measured plasma shapes, plasma parameters, the ionization equilibrium as discussed in the preceding section and estimated transport coefficients into account. The exact values of the latter do not matter within a certain plasma radius as discussed in section 2. For investigations of the atomic data, plasmas which exhibit a flat impurity profile have been chosen. These are identified by the radiation profile and the calibrated tungsten emissions (see [4] for the calibration of tungsten emissions) with an accuracy of about factor 2 .

The calculation results of an atomic code depends to a large extent on how accurate 

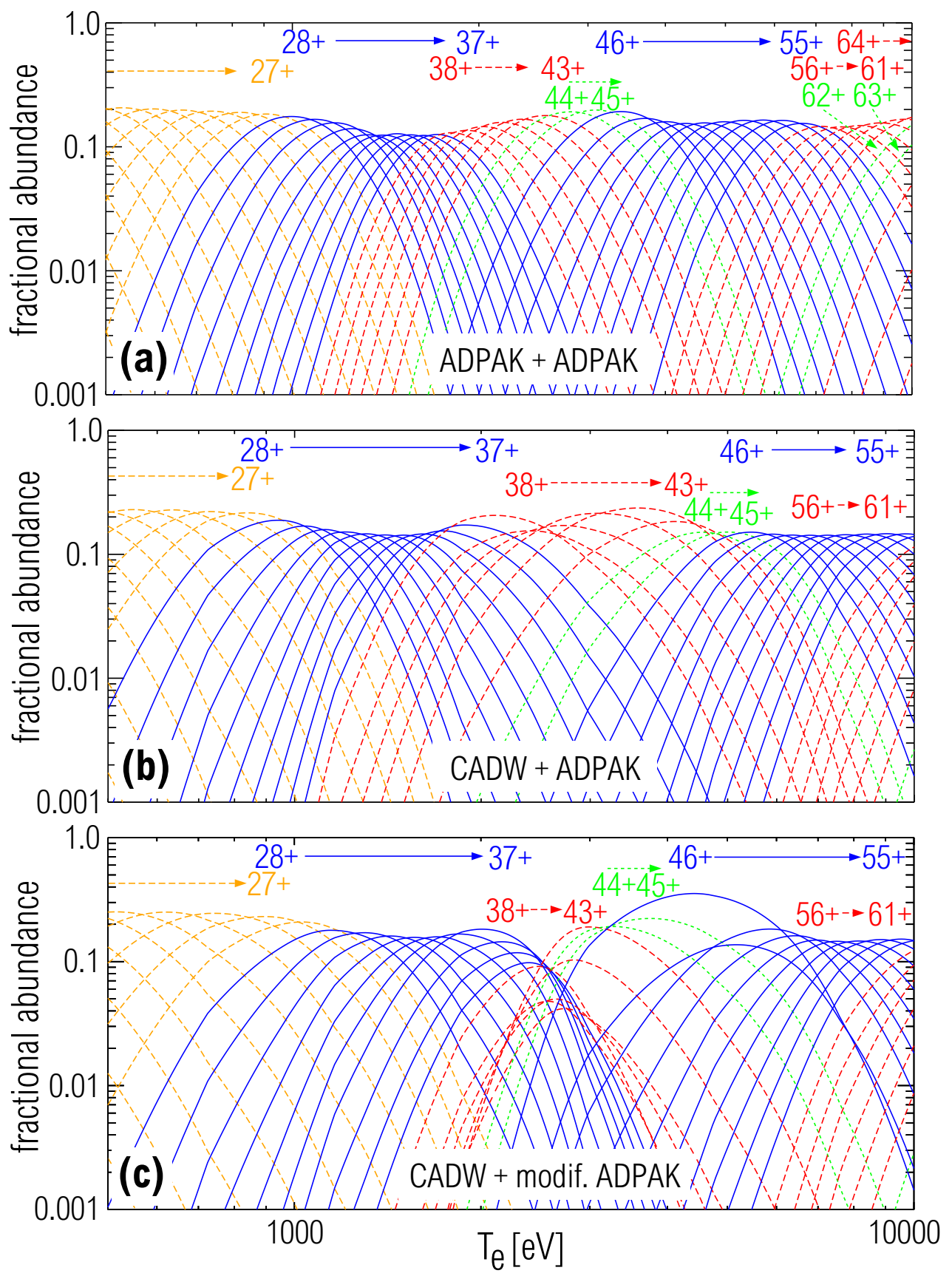

Figure 6. (a) Transport-free fractional abundances of tungsten ionization stages for zero electron density using ADPAK ionization and recombination data from [9], which were modified according to [4]. Numbers denote the ionization stage. Line styles and colors group ions with the same 'outermost' subshell; (b) Same as (a), but ionization rates taken from [32]; (c) Same as (b), while recombination rates have been modified (see table 1) according to experimental findings.

the wave functions are determined. Within the code, the wave functions are described using a linear combination of input wave functions (sets of input wave functions are specified by input configurations), such that the accuracy of the description depends on whether the right (or 

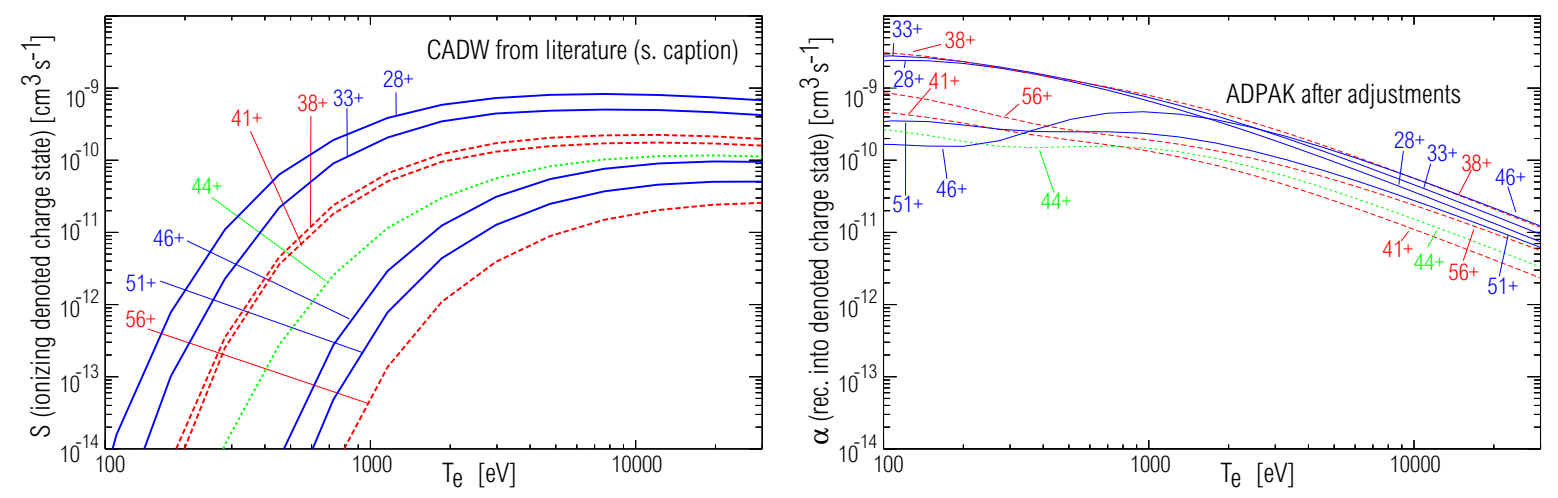

Figure 7. Ionization rates $S$ from [32] and recombination rates $\alpha$ obtained by modifying ADPAK data for several ionization stages of tungsten. Both data sets are used to obtain the ionization equilibrium labelled 'CADW+modif.ADPAK'. Colors and line styles correspond to those in figure 6

large enough) set of input wave functions was chosen for the calculation. As the plane wave Born approximation is a comparably fast method large sets of input configurations could be used. Determining the best choice of input configurations led to extensive testing on ionization stages above Cd-like $\mathrm{W}^{26+}$. For lower charge stages the number of levels were too large to arrive at an unambiguous configuration set. (for lower charges the calculations get very large, see below). For that purpose, the fast plane wave Born approximation is advantegous compared to other calculation methods like distorted-wave or $R$-matrix calculations. The results for electron impact cross sections at high electron energies are considered to be of good quality [15], while the more elaborate methods provide better quality at low electron energies. As a drawback, the plane wave Born approximation lacks the possibilty of spin changes during an electron collision, which might influence the resulting line strengths. However, this drawback is typically less important for high-Z elements, as the total spin quantum number is not well defined. The plane-wave Born approximation and the distorted wave calculations both lack the possibilty of taking resonances in the cross sections at low electron energies into account, which has impact on the excitation rates in a plasma. Unfortunately, $R$-matrix calculations, which could handle such effects consume a lot of computing time, such that only certain ionization stages can be targeted today [34, 35]. Another problem occurs for many ionization stages below Sn-like $\mathrm{W}^{24+}$, as open $4 \mathrm{f}$-shells occur, which leads to thousands of levels for relatively small set of input configurations (e.g. 3401 levels for I-like $\mathrm{W}^{21+}$ with $4 d^{10} 4 f^{6}$ and $4 d^{9} 4 f^{7}$ ). Even the version of the Cowan code, which was used in this work, could not perform the electron collision calculations with this vast amount of levels. Nevertheless, some of the ions with open $4 \mathrm{f}$-shells could be targeted by the Cowan code.

Bearing the above in mind, the implications for the following modelled spectra are that line intensities of single spectral lines might exhibit larger uncertainties (estimated to be ranging up to factors of about 2-3), but the overall intensity emitted by an ionization stage should be described more accurately, because deviations of calculated excitations cross sections should not be systematic. 


\section{Strong Spectral Features of Tungsten}

For diagnostic purposes a good signal to noise ratio is important. Especially in ITER or a reactor, $\mathrm{W}$ will be abundant in very small concentrations $\left(<10^{-4}\right.$ for managable radiative losses) and at the same time a considerable level of Bremsstrahlung and additionally neutron radiation will be abundant contributing to background noise. Therefore, the main focus is placed on strong spectral emissions.

\subsection{Spectral feature at $0.4-0.8 \mathrm{~nm}$}

Many spectral lines in this range have been identified earlier by $[5,6,8]$. In the present work, a comparison between the measured and modelled spectra is presented, while for the modelling the emissivity of spectral lines calculated by HULLAC [36] and the Cowan code is used. The ionization stages of Br-like $\mathrm{W}^{39+}$ to $\mathrm{Mn}$-like $\mathrm{W}^{49+}$ contribute considerable spectral lines in this range. The emissions of the $\mathrm{Ni}$-like $\mathrm{W}^{46+}$ are additionally compared using data from a $R$-matrix calculation [34]. Figure 8 presents the measured spectrum together with the modelled ones. The wavelength predictions of all calculations agree better than $0.002 \mathrm{~nm}$. The measured spectrum was obtained in a discharge with a tungsten concentration of about $2 \cdot 10^{-4}$, which is obtained by the method explained in [4]. The $\mathrm{W}$ concentration profile is flat, while a weak central impurity accumulation with impurity peaking of up to a factor of 3 in a very small central region cannot be excluded within the uncertainties. The comparison is performed by using this concentration in the modelling of all spectra. Most of the lines are modelled within a factor of 2 compared to the measurement. Differences between the models are small. A large difference between modelling and measurement is found at $0.793 \mathrm{~nm}$. The ADAS, HULLAC and $R$-matrix based models are too small by a factor of about 10, 3 and 3 , respectively. The HULLAC modelling would be further away than only a factor of 3 , if corrections due to excitation following inner-shell-ionization had not been included in the line intensity. In the $R$-matrix case, these contributions are neglected, however, the blending of a magnetic octopole line [37, 34] ([37] a distorted-wave calculation was performed and the data in [34] corresponds to the presented $R$-matrix modelling), is taken into account. In [37] the contribution from this line was more significant than in the modelled spectra in figure 8 possibly because the modelling was done for an monoenergetic electron beam at $4.06 \mathrm{keV}$, which results in a stronger magnetic octopole line compared to a Maxwellian electron velocity distribution. Even if both mentioned corrections are applied, the modelling cannot match up completely with the measured line intensity. For the modelling with ADAS data the described effects are neglected and additionally, a populating process from a higher (in energy) level identified in [37] is not taken into account. This is, because the plane wave Born approximation cannot be used to calculate the excitation of that higher level, as a spin changing transition is necessary to populate that level. Therefore, it is understood why the result of the ADAS calculations exhibits the largest discrepancies for this particular spectral line. Apart from this transition the Cowan code is a good model of the remaining lines and is therefore well suited to being used to generate spectra at baseline quality in a fast manner. 


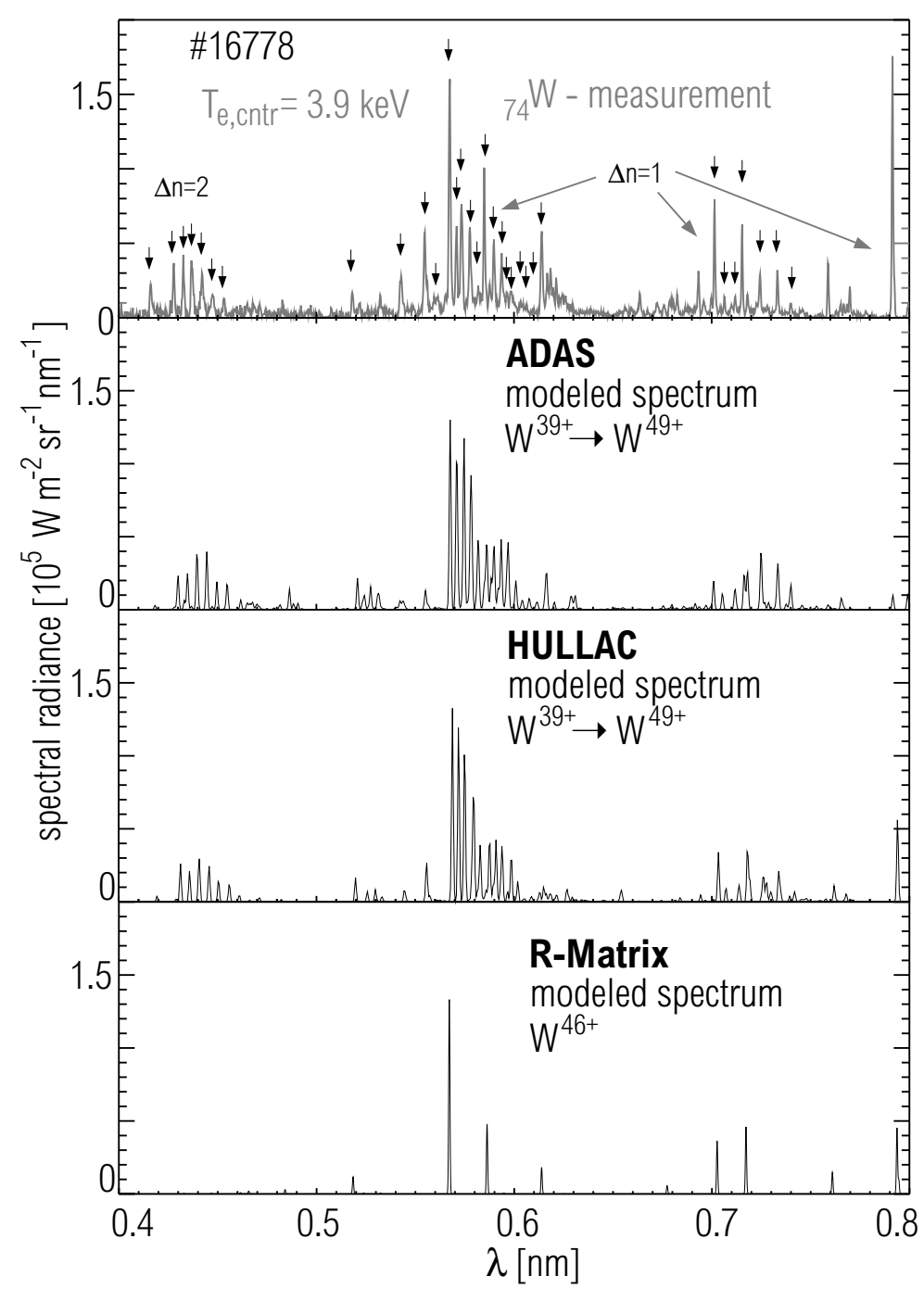

Figure 8. Strong spectral feature emitted by Br-like $\mathrm{W}^{39+}$ to about $\mathrm{Mn}$-like $\mathrm{W}^{49+}$ in the soft X-ray range (scanning Bragg crystal spectrometer). The measurement is compared to the modelling based on ADAS data (this work), HULLAC data [36] and $R$-matrix data [34] (only $\mathrm{W}^{46+}$ ) at a concentration of $2 \cdot 10^{-4}$. Small arrows indicate the spectral lines used in section 2 for quantifying fractional abundances of ionization stages.

\subsection{Quasicontinuum around $5 \mathrm{~nm}$}

In this spectral range the most intense feature emitted from ASDEX Upgrade plasmas is found which has been the focus of earlier investigations [2, 3, 11, 12, 14]. It consists of hundreds of spectral lines emitted by ionization stages from possibly as low charged ions as I-like $\mathrm{W}^{21+}$ up to about $\mathrm{Kr}$-like $\mathrm{W}^{38+}$, while the strongest emissions originate between Ag-like $\mathrm{W}^{27+}$ to Y-like $\mathrm{W}^{35+}$. In figure 9 (a), the spectral feature is presented along with the modelled result. A discharge with impurity accumulation is choosen to highlight the emissions at about $1.5 \mathrm{keV}$ electron temperature. The 'main' peak at $4.8 \mathrm{~nm}$ to about $5.4 \mathrm{~nm}$ is observed in the modelling exhibiting some difference in shape. These differences might be a result of the ionization balance, which could not be optimized to such detail. The influence of the 

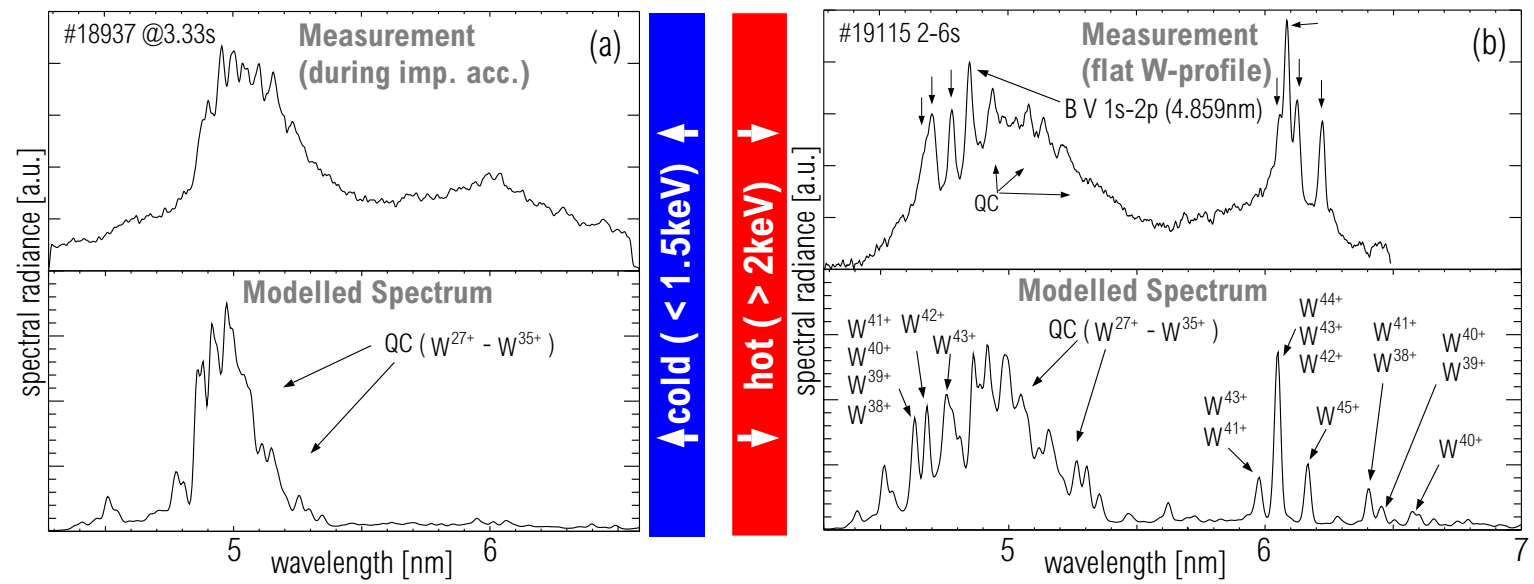

Figure 9. (a) Emissions of Ag-like $\mathrm{W}^{27+}-\mathrm{Y}$-like $\mathrm{W}^{35+}$ (grazing incidence spectrometer) during a discharge with impurity accumulation highlighting these ionization stages and modelled spectra using a peaked W-profile. (b) Same spectral range at higher $\mathrm{T}_{e}$ and flat W-profile, exhibiting additional spectral lines emitted by ionization stages up to $\mathrm{W}^{45+}$ and the corresponding modelling. The emissions depicted in part (a) are still visible because the LOS passes also through colder plasma at the plasma edge. Small arrows indicate the spectral lines used in section 2 for quantifying fractional abundances of ionization stages.

ionization balance is directly influencing the shape of the feature, because each ionization stage between $\mathrm{Rh}$-like $\mathrm{W}^{29+}$ to $\mathrm{Y}$-like $\mathrm{W}^{35+}$ contributes a spectral band consisting of many spectral lines with a width of about $0.15 \mathrm{~nm}$ to the quasicontinuum peak. For higher ion charges the emitted band is shifted to longer wavelengths. The compound emissions of all the ionization stages is forming the envelope of the quasicontinuum, which exhibits a width of about $0.5 \mathrm{~nm}$. This systematical dependence has been shown earlier [11, 14]. The wavelengths of the actual modelling agree within $0.05 \mathrm{~nm}$ with the calculations from [11] performed by the atomic code HULLAC [36]. However, the wavelengths measured in [11] exhibit discrepancies compared to the calculations. Additionally, the tokamak measurements can not be explained fully as a superposition of the measured spectra in [11]. Figure 10 presents the predicted and measured wavelengths and widths of the spectral feature from [11] and the present work, while the wavelength range of the whole quasicontinuum as measured in ASDEX Upgrade is also indicated. According to the latter, taking the EBIT measurements into account, the emissions between $4.85 \mathrm{~nm}$ and $5.05 \mathrm{~nm}$ in the tokamak spectrum should originate from Ag-like $\mathrm{W}^{27+}$ and Pd-like $\mathrm{W}^{28+}$. In the modelling emissions from these ionization stages are found, however, they do not fill the full wavelength range between $4.85 \mathrm{~nm}$ and $5.05 \mathrm{~nm}$ and a discrepancy remains. It should be noted, that the identification of these ionization stages from the EBIT measurements is not straightforward, as several ionization stages coexist in this energy range. This is the case, because the ionization potentials are separated by only $50-70 \mathrm{eV}$ for the ionization stages with several $3 \mathrm{~d}$-electrons in the ground state configuration. A further complication might arise, because the modelling also predicts emissions from ionization stages with lower charges than $\mathrm{Ag}$-like $\mathrm{W}^{27+}$ down to I-like $\mathrm{W}^{21+}$ with brightnesses of about the same level than for the above mentioned ionization 
stages. These predicted emissions are covering the full peak width from $4.8 \mathrm{~nm}$ to about $5.4 \mathrm{~nm}$ for each ionization stage, but have not been observed in the EBIT investigations. For the tokamak spectra a contribution from these ionization stages seems probable, because the emissions at $5 \mathrm{~nm}$ reach their maximum at about $1 \mathrm{keV}$ electron temperature, which can only be explained by a contribution from these lower charged ions or by drastic changes of ionization or recombination rates.

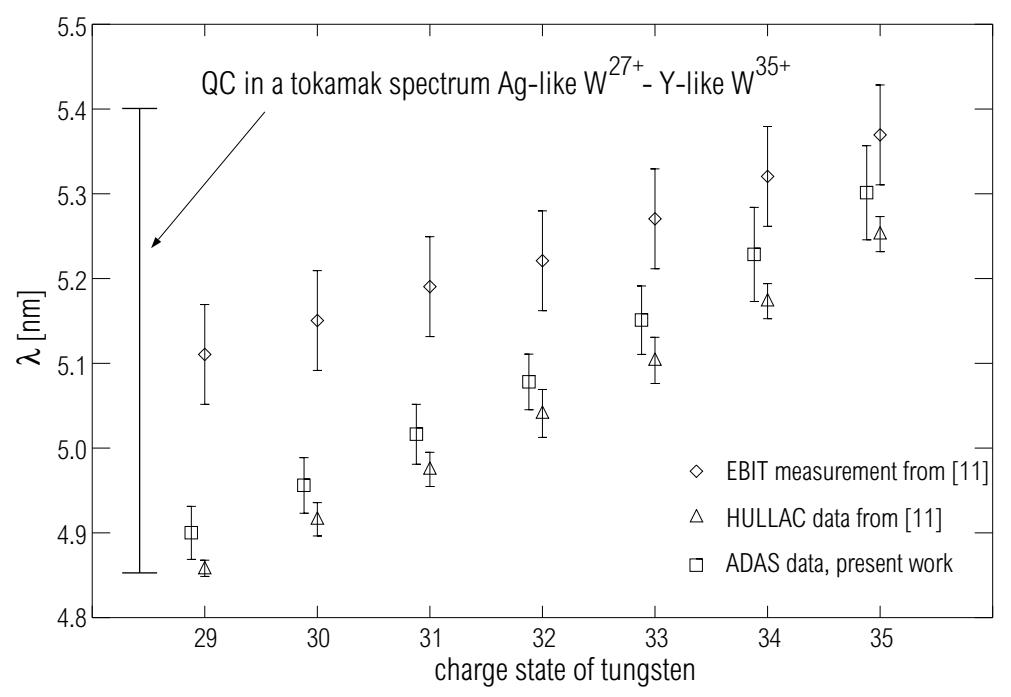

Figure 10. Wavelengths and spectral widths (indicated by bars) of the emissions responsible for the quasicontinuous structure around $5 \mathrm{~nm}$ from experiment and calculations. The ADAS data points (present work) have been displaced horizontally for better display.

At $5.7 \mathrm{~nm}$ to about $6.3 \mathrm{~nm}$ a less intense spectral feature is visible in the measurement, which is not seen to the same extent in the modelling. It is unclear, why this is the case, but as these emissions always coexist with the main peak of the quasicontinuum it is apparent that the emitting ionization stages are also in the range between I-like $\mathrm{W}^{21+}$ and Y-like $\mathrm{W}^{35+}$.

As reported earlier $[4,11,12,14]$ additional spectral lines are emitted by ionization stages of tungsten up to $\mathrm{Cu}$-like $\mathrm{W}^{45+}$ which are superimposed on the quasicontinuous emissions, when the central part of the plasma is hotter than about $2 \mathrm{keV}$. The quasicontinuum is still visible in the spectra, because the line of sight of the spectrometer is crossing colder parts of the plasma where W-ions with lower charge stages exist. In figure 9(b) this case is presented along with the modelling (note that this spectrum originates from a discharge with a flat $\mathrm{W}$ concentration profile). The intensity of spectral lines and quasicontinuous emissions agree relative to each other within the uncertainties. In agreement with the findings from [14], the structure at $4.7 \mathrm{~nm}$ can also be well understood by the modelled data as the Kr-like $\mathrm{W}^{38+}$ to about $\mathrm{W}^{41+}$ exhibit emissions with the right intensity and temperature dependence at this wavelength. It should be noted, that the wavelengths of modelled spectral lines in figure 9 result directly from the Cowan code calculations which is not fully relativistic but uses correction terms in the Hamiltonian, while for table 2 the theoretical wavelengths of many spectral lines have been recalculated by the fully relativistic code GRASP. This was 
done because in the VUV the discrepancies in wavelength between modelling using the Cowan code and measurement show up clearly being in the range of about $0.1-0.15 \mathrm{~nm}$ $(\Delta \lambda / \lambda \approx 1.5-3 \%)$ unlike for the SXR range where the discrepancies where below $0.002 \mathrm{~nm}$ $(\Delta \lambda / \lambda \approx 0.3 \%)$. A list of lines, which are well suited for diagnostics is given in table 2 . Additional line identifications can be found in $[4,11,12,14]$.

\subsection{Spectral Lines at $12-14 \mathrm{~nm}$}

In this wavelength range emissions of Se-like $\mathrm{W}^{40+}$ to $\mathrm{Cu}$-like $\mathrm{W}^{45+}$ are observed. In figure 11, a measured and modelled spectrum are compared. The line identification has been

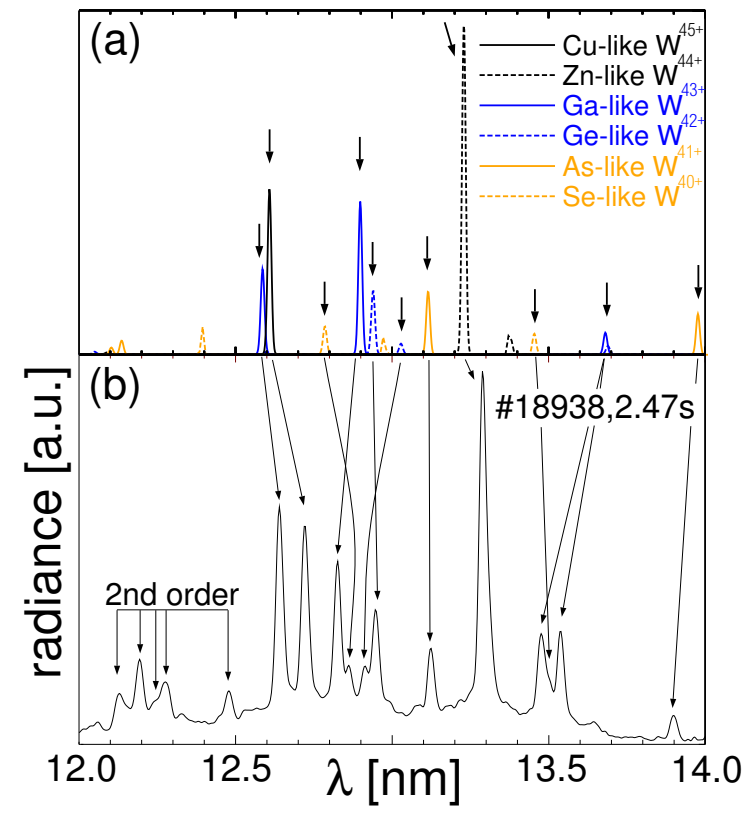

Figure 11. (a) Modelled W-emissions around $13 \mathrm{~nm}$. The emissivities of the spectral lines have been evaluated by ADAS calculations, while wavelengths have been corrected with the help of GRASP calculations afterwards. Small arrows indicate the spectral lines used in section 2 for quantifying fractional abundances of ionization stages. (b) Measured Wemissions (grazing incidence spectrometer) around $13 \mathrm{~nm}$. Line identifications can be found in $[14,13]$.

done in [14]. The wavelengths of the modelled lines are in this case corrected using the structure calculations of GRASP [38], which is a fully relativistic code. GRASP reproduces the wavelengths within an typical accuracy of $0.1-0.2 \mathrm{~nm}$ in this spectral range, which is better than the Cowan code, which exhibited discrepancies of up to $0.5 \mathrm{~nm}$. For diagnostic of tungsten in a fusion plasma, the spectral lines in this wavelength range are well suited, because several spectral lines are well isolated. However, there are tungsten emissions from plasma temperatures below $1 \mathrm{keV}$, which might blend with these spectral lines in special cases like described in the next subsection. 


\subsection{Quasicontinuum at $10-30 \mathrm{~nm}$}

At $10-30 \mathrm{~nm}$ many spectral lines overlap which are emitted from different ionization stages that exist below electron temperatures of about $1.3 \mathrm{keV}$. Similar to the quasicontinuum around $5 \mathrm{~nm}$, the emissions exhibit a dependence on electron temperature, which allows for deducing the emitting ionization stage. In figure 12(a), the time sequence after a W-injection by laser ablation is presented. Due to the fast rise of the emissions between $10-30 \mathrm{~nm}$ it is clear that the emitting ionization stages must be located in the plasma edge. A quantitative comparison of the timescales for the emissions between $10-30 \mathrm{~nm}$ and the emissions around $5 \mathrm{~nm}$ revealed that the emitting ionization stages of the first emissions are lower charged than $\mathrm{Pd}$ like $\mathrm{W}^{28+}$. In figure 12 (b) the emissions during impurity accumulation are detected from the central part of the plasma, while the central temperature is slowly dropping below $1.3 \mathrm{keV}$. Clearly, the emissions around $18 \mathrm{~nm}$ originate from the accumulation region possibly from the ionization stages Ag-like $\mathrm{W}^{27+}$ to about $\mathrm{Sn}$-like $\mathrm{W}^{24+}$. Just below $30 \mathrm{~nm}$ additional emissions are observed from the same ionization stages as deduced from the temporal behaviour of the spectral feature. The comparison of figure 12(a) and 12(b) implies that between about $19 \mathrm{~nm}$ and $27 \mathrm{~nm}$ spectral lines emitted at electron temperatures below $1.0 \mathrm{keV}$ can be found. These lines are not visible for electron temperatures above $1.0 \mathrm{keV}$ (figure 12(b)), while in figure figure 12(a) the emissions are visible after the laser ablation increased the tungsten density at the plasma edge, i.e. at electron temperatures from about $10 \mathrm{eV}$ to $1000 \mathrm{eV}$. The presented emissions are distributed over a wide spectral range. They are barely detectable in the spectrum, unless impurity accumulation occurs which cooles down the central accumulation region plasma to low enough temperatures, or $\mathrm{W}$ is injected into the plasma which inreases the $\mathrm{W}$-concentration at the edge drastically.

The modelling results presented in figure 12 support the experimental findings that the strong emissions around $18 \mathrm{~nm}$ originate from the ionization stages Cd-like $\mathrm{W}^{26+}$ to $\mathrm{Sn}$-like $\mathrm{W}^{24+}$, while the unaccounted emissions between $19 \mathrm{~nm}$ and $27 \mathrm{~nm}$ could be the emissions of Sb-like $\mathrm{W}^{23+}$ to $\mathrm{Nd}$-like $\mathrm{W}^{14+}$. Modelling is limited to the ionization stages above $\mathrm{Sn}$ like $\mathrm{W}^{24+}$. The atomic calculations are difficult for lower charged ionization stages, because the ground state of Sn-like $\mathrm{W}^{24+}$ is contained in the configuration $4 \mathrm{~d}^{10} 4 \mathrm{f}^{4}$, while the most important excited configuration is $4 \mathrm{~d}^{9} 4 \mathrm{f}^{5}$, yielding a total of 1985 levels. The emissions between $10-30 \mathrm{~nm}$ contain contributions from the configurations $4 \mathrm{f}^{3} 5 \mathrm{~s}, 4 \mathrm{f}^{3} 5 \mathrm{p}, 4 \mathrm{f}^{3} 5 \mathrm{~d}$ and $4 f^{3} 5 f$, while $4 f^{3} 5 \mathrm{~g}$ does not strongly influence the emissions in the considered spectral range. Given the boundary conditions of the available code, the calculations for lower charged ions was not possible to the same detail than for Cd-like $\mathrm{W}^{26+}$ to $\mathrm{Sn}$-like $\mathrm{W}^{24+}$.

\section{Additional Observations}

In many spectral regions, emissions of tungsten are apparent during impurity accumulation because comparably weak emissions get enhanced by this phenomena, such that the spectral lines rise above the limit of detection. In the following, some of these features are briefly reported. In figure 13(a), a spectral feature is presented, which could be identified in 


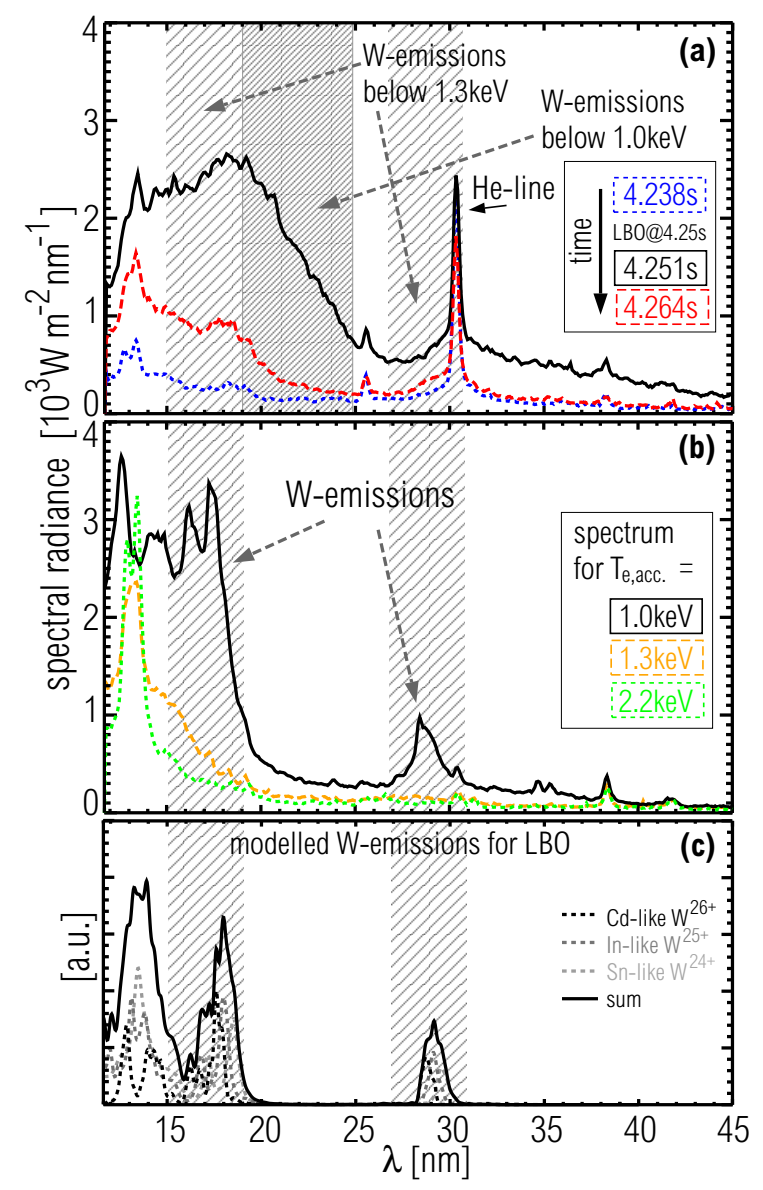

Figure 12. (a) Measured W-emissions (SPRED spectrometer) before and after W injection by laser ablation. The short time constant of the emissions demonstrates that the emissions take place at the very plasma edge. (b) Measured W-emissions (SPRED spectrometer) around $18 \mathrm{~nm}$ during impurity accumulation, where $\mathrm{T}_{e}$ in the accumulation region $\left(\mathrm{T}_{e, a c c .}\right)$ drops from $2.2 \mathrm{keV}$ to $1.0 \mathrm{keV}$. (c) Modelled W-emissions during a LBO, for a few ionization stages only, as calculations for lower ionization stages than Sn-like $\mathrm{W}^{24+}$ could not be performed in the necessary detail.

the modelling as being emitted by ionization stages below Mo-like $\mathrm{W}^{32+}$ down to Sn-like $\mathrm{W}^{24+}$. These emissions have already been reported and analyzed in [3, 39], while their behaviour versus electron temperature is documented in the present work. In figure 13, also the theoretical predictions of ADAS (intensity and wavelength) for each contributing ionization stage are presented, which originate from transitions between the configurations $4 \mathrm{~d}^{10} 4 \mathrm{f}^{n}$ and $4 \mathrm{~d}^{9} 4 \mathrm{f}^{n} 5 \mathrm{p} / 4 \mathrm{~d}^{10} 4 \mathrm{f}^{n-1} 5 \mathrm{~g}$ for ionization stages below Ag-like $\mathrm{W}^{27+}$ (inclusive) and between $4 \mathrm{~d}^{n}$ and $4 \mathrm{~d}^{n-1} 5 \mathrm{p}$ for the ionization stages above. For the ionization stages Rhlike $\mathrm{W}^{29+}$ to Tc-like $\mathrm{W}^{31+}$ also contributions from $4 \mathrm{~d}^{n}$ to $4 \mathrm{~d}^{n-1} 5 \mathrm{~s}$ are predicted between 2.9 and $3.5 \mathrm{~nm}$. The presented modelling assumes equal abundances of ionization stages. The wavelength-shift of the spectral feature for decreasing electron temperature represents a change of the ionization equilibrium. This direction of the wavelength shift is in agreement with increasing wavelengths of the predicted spectral lines for decreasing ionization stages. 


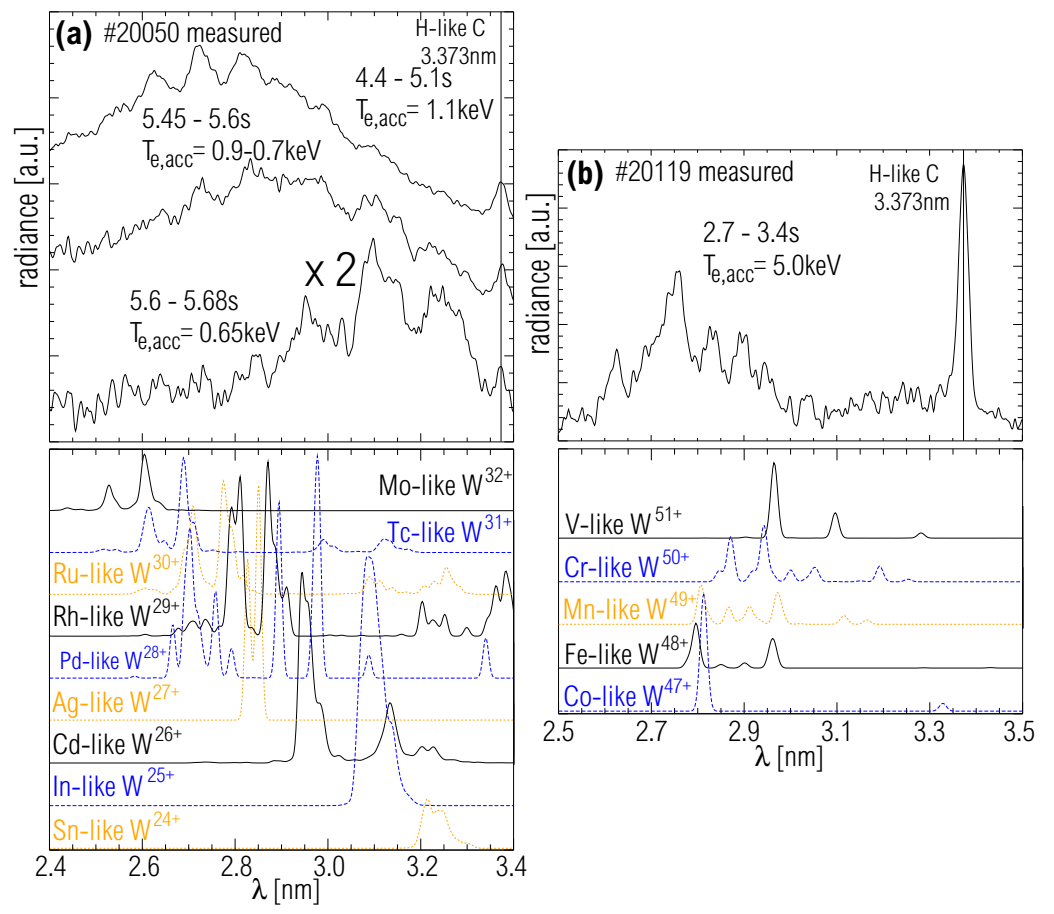

Figure 13. (a) Emissions at $\mathrm{T}_{e} \leq 1.1 \mathrm{keV}$ (grazing incidence spectrometer) during impurity accumulation together with ADAS modelling for corresponding ionization stages presented beneath. The measurement at $\mathrm{T}_{e, a c c}=0.65 \mathrm{keV}$ was scaled by factor of 2 . Each spectrum is shifted on the intensity axis for better visibility.(b) Emissions at $\mathrm{T}_{e} \approx$ $5 \mathrm{keV}$ (grazing incidence spectrometer) during impurity accumulation together with ADAS modelling presented beneath.
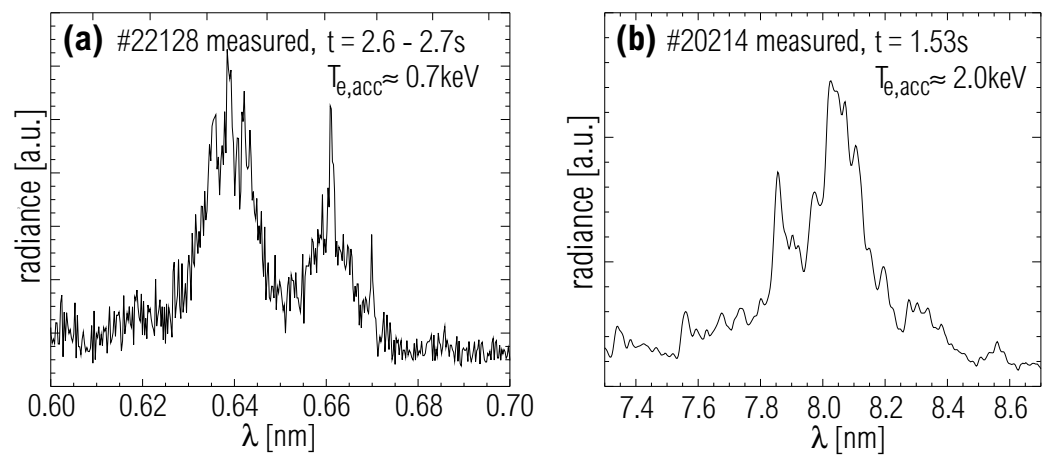

Figure 14. (a) Emissions at $\mathrm{T}_{e} \approx 0.7 \mathrm{keV}$ (scanning Bragg crystal spectrometer) during impurity accumulation. (b) Emissions at $\mathrm{T}_{e} \approx 2 \mathrm{keV}$ (grazing incidence spectrometer) during impurity accumulation.

In figure 13 (b), spectral lines of highly charged $\mathrm{W}$ are presented, which are observed in the same spectral range. The wavelengths of the modelling do not agree well enough to identify all spectral lines. The configurations, which are responsible for the modelled spectral lines are $3 \mathrm{p}^{6} 3 \mathrm{~d}^{n}$ and $3 \mathrm{p}^{5} 3 \mathrm{~d}^{n+1}$. These lines being emitted by ionization stages above Ni-like $\mathrm{W}^{46+}$ prove to be very important for diagnosing $\mathrm{W}$ in hot JET plasmas or in ITER, where Ni-like 
$\mathrm{W}^{46+}$ will only exist in the outer half of the plasma (see next section). In figure 14 (a) and (b) two features are depicted, which could not be found in the modelling. From the electron temperature range, in which they have been emitted, conclusions about the emitting ionization stage can be drawn. The emissions in figure 14 (a) are emitted by ionization stages well below Sr-like $\mathrm{W}^{36+}$ as the electron temperature of $0.7 \mathrm{keV}$ suggests the abundance of I-like $\mathrm{W}^{21+}$ to about Pd-like $\mathrm{W}^{28+}$. The photon energy $(\geq 1.85 \mathrm{keV})$ for the presented emissions is more than the ionization potential (for Sr-like $\mathrm{W}^{36+}$ the ionization potential is $1.591 \mathrm{keV}$ according to [40]) of the abundant ionization stages. Therefore, this feature is possibly emitted during the process of dielectronic recombination, which is not included in the spectral modelling. Figure 14 (b) depicts $\mathrm{W}$ emissions at $8 \mathrm{~nm}$, which are emitted by ionization stages between Sr-like $\mathrm{W}^{36+}$ and $\mathrm{Zn}$-like $\mathrm{W}^{44+}$.

\section{Predictions for ITER}

In figure 15, the modelling predictions for a typical ASDEX Upgrade and ITER plasma are presented. While in the right column the fractional abundances of $\mathrm{W}$ ionization stages are depicted, the modelled spectra are presented on the left. In the case of ASDEX Upgrade a H-mode discharge with average performance was chosen (central electron parameters are given in the figure). The ITER case is based on the calculated electron profiles in [41]. A radial viewing geometry at the midplane is used for the modelled spectra. Absolute numbers are given for the spectral brigthness. The modelled results of ASDEX Upgrade depicted in figure 15 reproduce the most important spectral features observed in experiment (see above). In ITER, central electron temperatures of $18 \mathrm{keV}$ and above (e.g. advanced scenarios) are envisaged. The current experience with $\mathrm{W}$ spectroscopy provides an insight into the outer part of an ITER plasma. An appraisal of the full diagnostic capability of spectroscopic measurements of $\mathrm{W}$ relies on models. The predictions are intended to demonstrate the diagnostic needs for such a plasma, while the exact weighting of ionization states and line intensities contain larger uncertainties than the modelling for lower electron temperatures as less experimental background information is available. For plasma transport a rough estimation was used, which is $0.5 \mathrm{~m}^{2} / \mathrm{s}$ increasing to $2 \mathrm{~m}^{2} / \mathrm{s}$ from the core plasma to the pedestal top for the diffusion coefficient. The drift velocity was adjusted such that a constant $\mathrm{W}$ concentration is found inside the pedestal top. The diffusion coefficient and drift velocity are chosen such that their magnitude is comparable with the findings of actual tokamaks. This is an assumption, because there is no precise prediction for ITER and it is beyond the scope of this work to provide detailed transport coefficents. However, for the ionization equilibrium in the confined plasma, the large ionization and recombination rates make plasma transport unimportant compared to low-Z impurities. Apart from the assumptions on the shape of the $\mathrm{W}$ concentration profile, the $\mathrm{W}$ source was adjusted such that an ITER-relevant $\mathrm{W}$ concentration of $10^{-5}$ is found in the plasma. From mid-radius to the plasma center $\mathrm{Cr}$-like $\mathrm{W}^{50+}$ to $\mathrm{C}$ like $\mathrm{W}^{68+}$ or maybe for special discharges even He-like $\mathrm{W}^{72+}$ might exist in a diagnosable abundance. To allow for a possible extrapolation the ionization equilibrium for electron temperatures between $5 \mathrm{keV}$ and $40 \mathrm{keV}$ is presented in figure 16. In this temperature range, 


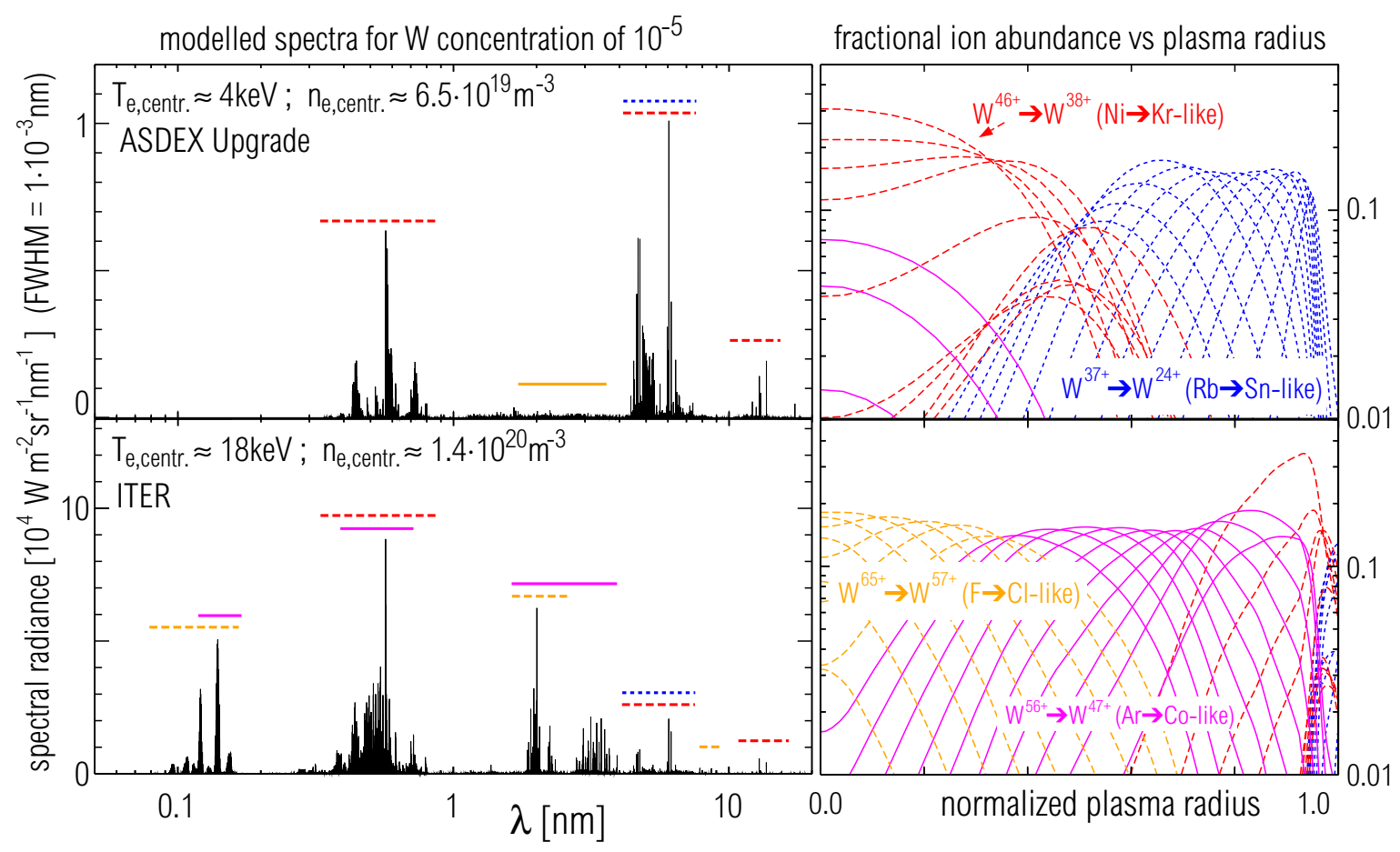

Figure 15. Modelling results and predictions for a typical ASDEX Upgrade plasma and the standard ITER scenario. In the left column the predicted spectra are presented. The fractional abundances of ionization stages vs. normalized plasma radius $\left(\rho_{\text {pol }}\right)$ are depicted in the right column. The horizontal lines on the left indicate the emitting ionization stages by the line style and brightness, corresponding to the line style and brightness in the right column.

the adjustments of the ionization equilibrium performed in section 2 do not play a significant role. Hence, the equilibrium probably exhibits deviations from experiments. Already for the upcoming ITER-like wall project [42] at JET, the diagnosis of $\mathrm{W}$ at electron temperatures above $6 \mathrm{keV}$ will be of interest which requires the measurement and interpretation of the emissions from ionization stages above $\mathrm{Cr}$-like $\mathrm{W}^{50+}$.

In detail, the interesting wavelength regions for diagnosing the ionization stages between Co-like $\mathrm{W}^{47+}$ to Ar-like $\mathrm{W}^{56+}$ are the ranges $0.1-0.15 \mathrm{~nm}, 0.4-0.7 \mathrm{~nm}$ and $2.7-4.0 \mathrm{~nm}$. For the ionization stages $\mathrm{Cl}$-like $\mathrm{W}^{57+}$ to $\mathrm{W}^{70+}$ the wavelength ranges $0.1-0.15 \mathrm{~nm}, 1.8-2.4 \mathrm{~nm}$ and the region around $8 \mathrm{~nm}$ are of interest. Therefore, it seems that especially the ranges at $0.1-0.15 \mathrm{~nm}$ and at $1.8-4.0 \mathrm{~nm}$ are capable to provide information about tungsten from mid-radius to the center of the ITER plasma. These findings should be verified and quantified by performing dedicated experiments in fusion plasmas and also in EBIT devices, while the theoretical effort should be focussed on improving the accuracy of the key diagnostic stages.

\section{Spectral Emissions Suited for Diagnostics}

In table 2, an overview on recommended diagnostic lines is given, which have been verified by experiments. This table is not a complete list, but contains promising candidates 


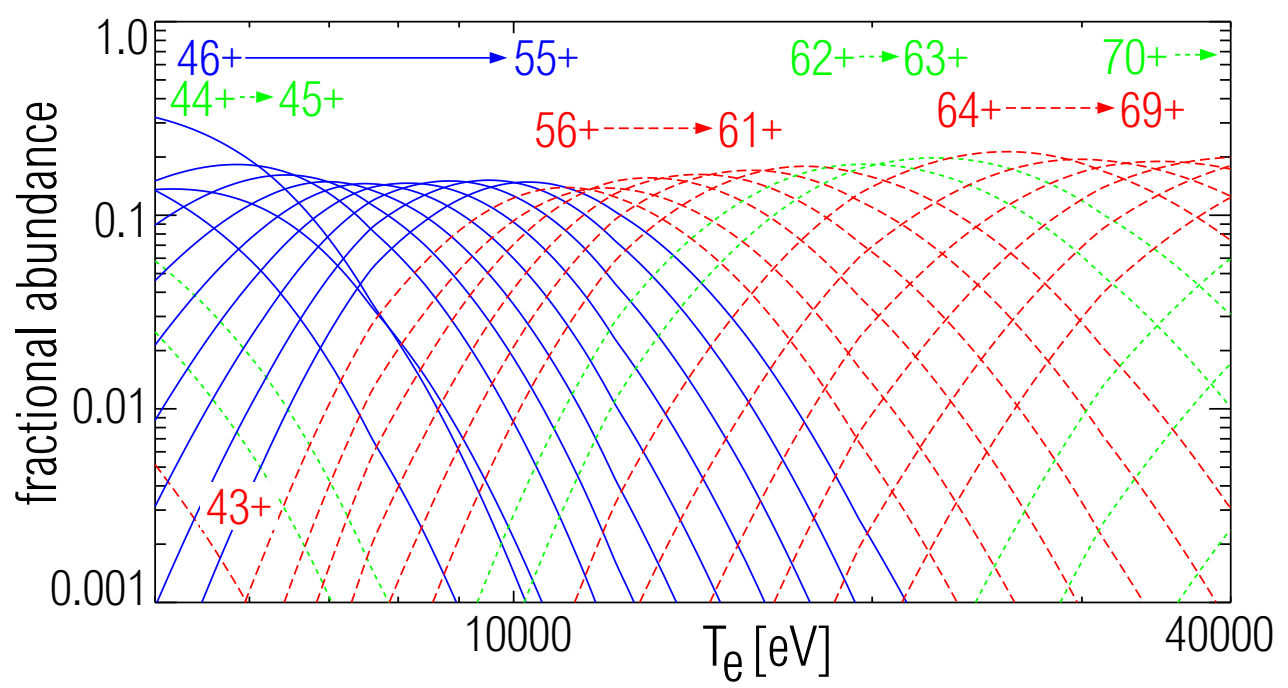

Figure 16. Transport-free ionization equilibrium for electron temperatures between $5 \mathrm{keV}$ and $40 \mathrm{keV}$ at zero density using ionization rates from [32] and ADPAK recombination rates, which have been modified according to the experimental findings ('CADW+modif. ADPAK', see table 1).

for diagnosing tungsten at various electron temperatures, i.e. different ionization stages. Calculated and measured wavelengths, configurations and terms are given. In cases, where no LS-coupled term contributes a major part of the wavefunction (less than 50\%) the J values and the transition probability A is given to identify the transition. To indicate possible problems with line blending the last column was introduced, in which B and BL are denoted for line blending of ionization stages of similar/same charge (B) and considerably lower charge (BL). If an additional ' $*$ ' is specified, the line blending is more severe. It may be noted that problems of line blending depend on the specifications of spectrometer hardware - therefore, the given indications may have to be reconsidered for different hardware. In table 3 predictions of spectral lines are listed, which look promising for future diagnostics in JET, ITER or a reactor. For these predictions, also a column is given for anticipated line blending, derived from modelling, which assumed similar hardware than present on ASDEX Upgrade. In that respect, the emissions at $0.1-0.15 \mathrm{~nm}$ seem to be well suited for central tungsten diagnostics, as emissions from lower charged ions are not blended with the spectral lines.

\section{Summary}

Detailed experimental investigations on W spectra at ASDEX Upgrade along with modelling efforts using fundamental atomic data calculations have been performed to provide more insight in the characteristics of the spectral emissions of W. Recommendations for quantitatively diagnosing tungsten at various electron temperatures in fusion plasmas and an overview of spectral features found in the spectra of tungsten have been presented. Spectroscopic measurements during the transport phenomenon of impurity accumulation 


\begin{tabular}{|c|c|c|c|c|c|}
\hline Ion & I.P. $(\mathrm{eV})$ & $\overline{\lambda_{\text {calc }}(\mathrm{nm})}$ & $\lambda_{t o k}(\mathrm{~nm})$ & transition/configurations & blend. \\
\hline $\begin{array}{l}\approx \mathrm{W}^{14+}-\approx \mathrm{W}^{28+} \\
(\mathrm{Nd}-\text { like }- \text { Pd-like })\end{array}$ & $\begin{array}{l}325.3- \\
1132\end{array}$ & $10-30$ & $10-30$ & many (cf. text) & $\mathrm{B}$ \\
\hline $\begin{array}{l}\approx \mathrm{W}^{21+}-\mathrm{W}^{35+} \\
(\mathrm{I}-\text { like }- \text { Sr-like })\end{array}$ & $\begin{array}{l}594.5- \\
1512\end{array}$ & $4.5-6.5$ & $4.5-6.5$ & $\begin{array}{c}\leq \mathrm{W}^{28+}: 4 \mathrm{~d}^{9} 4 \mathrm{f}^{n+1}-4 \mathrm{~d}^{10} 4 \mathrm{f}^{n} \\
>\mathrm{W}^{28+}:\left(4 \mathrm{p}^{6} 4 \mathrm{~d}^{n-1} 4 \mathrm{f}\right) /\left(4 \mathrm{p}^{5} 4 \mathrm{~d}^{n+1}\right)- \\
-4 \mathrm{p}^{6} 4 \mathrm{~d}^{n}\end{array}$ & $\begin{array}{l}\mathrm{B} \\
\mathrm{B}\end{array}$ \\
\hline $\begin{array}{l}\mathrm{W}^{39+} \\
\text { (Br-like) }\end{array}$ & 1883 & $6.403(\mathrm{G})$ & 6.457 & $4 s^{2} 4 p^{5}{ }^{2} P_{3 / 2}-4 s^{2} 4 p^{4} 4 d^{2} F_{5 / 2}$ & BL* \\
\hline $\begin{array}{l}\mathrm{W}^{40+} \\
\text { (Se-like) }\end{array}$ & 1941 & $\begin{array}{r}6.243(\mathrm{G}) \\
12.786(\mathrm{G}) \\
13.455(\mathrm{G})\end{array}$ & $\begin{array}{r}6.268 \\
12.864 \\
13.487\end{array}$ & $\begin{array}{l}4 s^{2} 4 p^{4}{ }^{3} \mathrm{P}_{2}-4 s 4 \mathrm{p}^{5}{ }^{3} \mathrm{P}_{2} \\
4 \mathrm{~s}^{2} 4 \mathrm{p}^{4}{ }^{3} \mathrm{P}_{2}-4 \mathrm{~s}^{2} 4 \mathrm{p}^{4}{ }^{1} \mathrm{D}_{2} \\
4 \mathrm{~s}^{2} 4 \mathrm{p}^{4}{ }^{3} \mathrm{P}_{2}-4 \mathrm{~s}^{2} 4 \mathrm{p}^{4}{ }^{3} \mathrm{P}_{1}\end{array}$ & $\begin{array}{l}\mathrm{BL}^{*} \\
\mathrm{~B}^{*} \\
\mathrm{~B}^{*}\end{array}$ \\
\hline $\begin{array}{l}\mathrm{W}^{41+} \\
\text { (As-like) }\end{array}$ & 1995 & $\begin{array}{r}6.482(\mathrm{G}) \\
13.106(\mathrm{G}) \\
13.968(\mathrm{G})\end{array}$ & $\begin{array}{r}6.481 \\
13.121 \\
13.896\end{array}$ & $\begin{array}{l}4 s^{2} 4 p^{32} D_{3 / 2}-4 s 4 p^{4}{ }^{4} P_{5 / 2} \\
4 s^{2} 4 p^{3}{ }^{2} D_{3 / 2}-4 s^{2} 4 p^{3}{ }^{2} D_{5 / 2} \\
4 s^{2} 4 p^{3}{ }^{2} D_{3 / 2}-4 s^{2} 4 p^{3}{ }^{4} S_{3 / 2}\end{array}$ & BL* \\
\hline $\begin{array}{l}\mathrm{W}^{42+} \\
\text { (Ge-like) }\end{array}$ & 2149 & $\begin{array}{r}0.5818(\mathrm{C}) \\
0.5823(\mathrm{C}) \\
0.6010(\mathrm{C}) \\
\\
4.685(\mathrm{G}) \\
6.115(\mathrm{G}) \\
12.940(\mathrm{G}) \\
13.029(\mathrm{G}) \\
13.690(\mathrm{G})\end{array}$ & $\begin{array}{r}0.583 \\
0.583 \\
0.601 \\
\\
4.718 \\
6.123 \\
12.945 \\
12.912 \\
13.475\end{array}$ & $\begin{array}{c}3 \mathrm{~d}^{10} 4 \mathrm{~s}^{2} 4 \mathrm{p}^{2}{ }^{3} \mathrm{P}_{0}-3 \mathrm{~d}^{9} 4 \mathrm{~s}^{2} 4 \mathrm{p}^{2} 4 \mathrm{f} J=1 \\
\left(A=2.5 \cdot 10^{14} s^{-1}\right) \\
3 \mathrm{~d}^{10} 4 \mathrm{~s}^{2} 4 \mathrm{p}^{2}{ }^{3} \mathrm{P}_{0}-3 \mathrm{~d}^{9} 4 \mathrm{~s}^{2} 4 \mathrm{p}^{2} 4 \mathrm{f} J=1 \\
\left(A=1.5 \cdot 10^{14} s^{-1}\right) \\
3 \mathrm{~d}^{10} 4 \mathrm{~s}^{2} 4 \mathrm{p}^{2}{ }^{3} \mathrm{P}_{0}-3 \mathrm{~d}^{9} 4 \mathrm{~s}^{2} 4 \mathrm{p}^{2} 4 \mathrm{f} J=1 \\
\left(A=1.3 \cdot 10^{14} s^{-1}\right) \\
4 \mathrm{~s}^{2} 4 \mathrm{p}^{2}{ }^{3} \mathrm{P}_{0}-4 \mathrm{~s}^{2} 4 \mathrm{p}^{4} \mathrm{~d}^{3} \mathrm{D}_{1} \\
4 \mathrm{~s}^{2} 4 \mathrm{p}^{2}{ }^{3} \mathrm{P}_{0}-4 \mathrm{~s} 4 \mathrm{p}^{3}{ }^{3} \mathrm{D}_{1} \\
4 \mathrm{~s}^{2} 4 \mathrm{p}^{2}{ }^{3} \mathrm{P}_{0}-4 \mathrm{~s}^{2} 4 \mathrm{p}^{2}{ }^{1} \mathrm{D}_{2} \\
4 \mathrm{~s}^{2} 4 \mathrm{p}^{2}{ }^{3} \mathrm{P}_{1}-4 \mathrm{~s} 4 \mathrm{p}^{3}{ }^{3} \mathrm{P}_{2} \\
4 \mathrm{~s}^{2} 4 \mathrm{p}^{2}{ }^{1} \mathrm{D}_{2}-4 \mathrm{~s} 4 \mathrm{p}^{3}{ }^{3} \mathrm{P}_{2}\end{array}$ & $\begin{array}{l}\text { B } \\
\text { B } \\
\text { B } \\
\mathrm{B}^{*}, \mathrm{BL} \\
\mathrm{B}^{*}, \mathrm{BL} \\
\mathrm{B} \\
\mathrm{B} \\
\mathrm{B}\end{array}$ \\
\hline $\begin{array}{l}\mathrm{W}^{43+} \\
\text { (Ga-like) }\end{array}$ & 2210 & $\begin{array}{l}0.5798(\mathrm{G}) \\
0.5801(\mathrm{G}) \\
0.5988(\mathrm{G}) \\
0.5989(\mathrm{G}) \\
4.760(\mathrm{G}) \\
6.020(\mathrm{G}) \\
6.119(\mathrm{G}) \\
12.587(\mathrm{G}) \\
12.899(\mathrm{G}) \\
13.682(\mathrm{G})\end{array}$ & $\begin{array}{r}0.579 \\
0.579 \\
0.598 \\
\\
0.598 \\
\\
4.791 \\
6.063 \\
6.135 \\
12.639 \\
12.824 \\
13.534 \\
\end{array}$ & $\begin{array}{c}3 \mathrm{~d}^{10} 4 \mathrm{~s}^{2} 4 \mathrm{p}^{2} \mathrm{P}_{1 / 2}-3 \mathrm{~d}^{9} 4 \mathrm{~s}^{2} 4 \mathrm{p} 4 \mathrm{f} J=\frac{1}{2} \\
\left(A=3.7 \cdot 10^{14} s^{-1}\right) \\
3 \mathrm{~d}^{10} 4 \mathrm{~s}^{2} 4 \mathrm{p}^{2} \mathrm{P}_{1 / 2}-3 \mathrm{~d}^{9} 4 \mathrm{~s}^{2} 4 \mathrm{p} 4 \mathrm{f} J=\frac{3}{2} \\
\left(A=4.0 \cdot 10^{14} s^{-1}\right) \\
3 \mathrm{~d}^{10} 4 \mathrm{~s}^{2} 4 \mathrm{p}^{2} \mathrm{P}_{1 / 2}-3 \mathrm{~d}^{9} 4 \mathrm{~s}^{2} 4 \mathrm{p} 4 \mathrm{f} J=\frac{3}{2} \\
\left(A=1.3 \cdot 10^{14} s^{-1}\right) \\
3 \mathrm{~d}^{10} 4 \mathrm{~s}^{2} 4 \mathrm{p}^{2} \mathrm{P}_{1 / 2}-3 \mathrm{~d}^{9} 4 \mathrm{~s}^{2} 4 \mathrm{p} 4 \mathrm{f} J=\frac{1}{2} \\
\left(A=1.3 \cdot 10^{14} s^{-1}\right) \\
4 \mathrm{~s}^{2} 4 \mathrm{p}^{2} \mathrm{P}_{1 / 2}-4 \mathrm{~s}^{2} 4 \mathrm{~d}^{2} \mathrm{D}_{3 / 2} \\
4 \mathrm{~s}^{2} 4 \mathrm{p}^{2} \mathrm{P}_{1 / 2}-4 \mathrm{~s} 4 \mathrm{p}^{2}{ }^{2} \mathrm{P}_{1 / 2} \\
4 \mathrm{~s}^{2} 4 \mathrm{p}^{2} \mathrm{P}_{1 / 2}-4 \mathrm{~s} 4 \mathrm{p}^{2}{ }^{2} \mathrm{D}_{3 / 2} \\
4 \mathrm{~s}^{2} 4 \mathrm{p}^{2} \mathrm{P}_{1 / 2}-4 \mathrm{~s}^{2} 4 \mathrm{p}^{2} \mathrm{P}_{3 / 2} \\
4 \mathrm{~s}^{2} 4 \mathrm{p}^{2} \mathrm{P}_{1 / 2}-4 \mathrm{~s} 4 \mathrm{p}^{2}{ }^{4} \mathrm{P}_{1 / 2} \\
4 \mathrm{~s}^{2} 4 \mathrm{p}^{2} \mathrm{P}_{3 / 2}-4 \mathrm{~s} 4 \mathrm{p}^{2}{ }^{2} \mathrm{D}_{5 / 2}\end{array}$ & $\begin{array}{l}\text { B } \\
\text { B } \\
\text { B } \\
\text { B } \\
\text { BL } \\
\text { B }^{*}, \text { BL } \\
\text { B }^{*}, \mathrm{BL} \\
\text { B }\end{array}$ \\
\hline $\begin{array}{l}\mathrm{W}^{44+} \\
\text { (Zn-like) } \\
\text { continued }\end{array}$ & 2355 & $\begin{array}{r}0.5749(\mathrm{G}) \\
0.5938(\mathrm{G}) \\
6.073(\mathrm{G}) \\
13.230(\mathrm{G})\end{array}$ & $\begin{array}{r}0.575 \\
0.595 \\
6.093 \\
13.287\end{array}$ & $\begin{array}{c}3 \mathrm{~d}^{10} 4 \mathrm{~s}^{2}{ }^{1} \mathrm{~S}_{0}-3 \mathrm{~d}^{9} 4 \mathrm{~s}^{2} 4 \mathrm{f}^{1} \mathrm{P}_{1} \\
3 \mathrm{~d}^{10} 4 \mathrm{~s}^{2}{ }^{1} \mathrm{~S}_{0}-3 \mathrm{~d}^{9} 4 \mathrm{~s}^{2} 4 \mathrm{f}^{3} \mathrm{D}_{1} \\
4 \mathrm{~s}^{2}{ }^{1} \mathrm{~S}_{0}-4 \mathrm{~s} 4 \mathrm{p}{ }^{1} \mathrm{P}_{1} \\
4 \mathrm{~s}^{2}{ }^{2} \mathrm{~S}_{0}-4 \mathrm{~s} 4 \mathrm{p}^{3} \mathrm{P}_{1}\end{array}$ & $\mathrm{~B}, \mathrm{BL}$ \\
\hline
\end{tabular}




\begin{tabular}{|c|c|c|c|c|c|}
\hline Ion & I.P. $(\mathrm{eV})$ & $\overline{\lambda_{\text {calc }}(\mathrm{nm})}$ & $\lambda_{t o k}(\mathrm{~nm})$ & transition/configurations & blend. \\
\hline \multirow{11}{*}{$\begin{array}{l}\mathrm{W}^{45+} \\
(\mathrm{Cu}-\text { like })\end{array}$} & \multirow[t]{11}{*}{2414} & $0.5721(\mathrm{G})$ & 0.572 & $3 d^{10} 4 s^{2} S_{1 / 2}-3 d^{9} 4 s 4 f^{2} P_{1 / 2}$ & $\bar{B}$ \\
\hline & & $0.5725(\mathrm{G})$ & 0.572 & $3 \mathrm{~d}^{10} 4 \mathrm{~s}^{2} \mathrm{~S}_{1 / 2}-3 \mathrm{~d}^{9} 4 \mathrm{~s} 4 \mathrm{f} J=\frac{1}{2}$ & B \\
\hline & & & & $\left(A=4.1 \cdot 10^{14} s^{-1}\right)$ & \\
\hline & & $0.5911(\mathrm{G})$ & 0.591 & $3 \mathrm{~d}^{10} 4 \mathrm{~s}^{2} \mathrm{~S}_{1 / 2}-3 \mathrm{~d}^{9} 4 \mathrm{~s} 4 \mathrm{f} J=\frac{1}{2}$ & B \\
\hline & & $0.010(C)$ & $0=501$ & $\left(A=1.3 \cdot 10^{14} s^{-1}\right)$ & \\
\hline & & $0.5912(\mathrm{G})$ & 0.591 & $\begin{array}{c}3 \mathrm{~d}^{10} 4 \mathrm{~s}^{2} \mathrm{~S}_{1 / 2}-3 \mathrm{~d}^{9} 4 \mathrm{~s} 4 \mathrm{f} J=\frac{3}{2} \\
\left(A=1.3 \cdot 10^{14} s^{-1}\right)\end{array}$ & B \\
\hline & & $0.7268(\mathrm{G})$ & 0.725 & $3 d^{10} 4 s^{2} S_{1 / 2}-3 d^{9} 4 s 4 p^{4} D_{1 / 2}$ & B \\
\hline & & $0.7273(\mathrm{G})$ & 0.725 & $3 d^{10} 4 s^{2} S_{1 / 2}-3 d^{9} 4 s 4 p J=\frac{3}{2}$ & B \\
\hline & & & & $\left(A=4.7 \cdot 10^{12} s^{-1}\right)$ & \\
\hline & & $6.217(\mathrm{G})$ & 6.232 & $4 s^{2} S_{1 / 2}-4 p^{2} P_{3 / 2}$ & BL \\
\hline & & $12.609(\mathrm{G})$ & 12.720 & $4 s^{2} \mathrm{~S}_{1 / 2}-4 \mathrm{p}^{2} \mathrm{P}_{1 / 2}$ & \\
\hline \multirow{5}{*}{$\begin{array}{l}\mathrm{W}^{46+} \\
(\mathrm{Ni}-\text { like })\end{array}$} & \multirow[t]{5}{*}{4057} & $0.5687(\mathrm{G})$ & 0.569 & $3 d^{10{ }^{1}} S_{0}-3 d^{9} 4 f^{1} P_{1}$ & \\
\hline & & $0.5875(\mathrm{G})$ & 0.587 & $3 d^{10}{ }^{1} S_{0}-3 d^{9} 4 f^{3} D_{1}$ & \\
\hline & & $0.7035(\mathrm{G})$ & 0.702 & $3 d^{10}{ }^{1} S_{0}-3 d^{9} 4 p^{1} P_{1}$ & \\
\hline & & $0.7184(\mathrm{G})$ & 0.716 & $3 d^{10}{ }^{1} S_{0}-3 d^{9} 4 p^{3} D_{1}$ & \\
\hline & & $0.7944(\mathrm{G})$ & 0.793 & $3 d^{10{ }^{1}} S_{0}-3 d^{9} 4 s{ }^{1} D_{2}$ & \\
\hline \multirow[t]{2}{*}{$\begin{array}{l}\mathrm{W}^{47+} \\
\text { (Co-like) }\end{array}$} & \multirow[t]{2}{*}{4180} & $0.5550(\mathrm{C})$ & 0.556 & $\begin{array}{c}3 \mathrm{~d}^{9}{ }^{2} \mathrm{D}_{5 / 2}-3 \mathrm{~d}^{8} 4 \mathrm{f} J=\frac{5}{2} \\
\left(A=2.3 \cdot 10^{14} s^{-1}\right)\end{array}$ & $\mathrm{B}$ \\
\hline & & $0.5553(\mathrm{C})$ & 0.556 & $\begin{array}{c}3 \mathrm{~d}^{9}{ }^{2} \mathrm{D}_{5 / 2}-3 \mathrm{~d}^{8} 4 \mathrm{f} J=\frac{7}{2} \\
\left(A=4.2 \cdot 10^{14} s^{-1}\right)\end{array}$ & $\mathrm{B}$ \\
\hline \multirow[t]{3}{*}{$\begin{array}{l}\mathrm{W}^{48+} \\
(\mathrm{Fe}-\text { like })\end{array}$} & \multirow[t]{3}{*}{4309} & $0.5438(\mathrm{C})$ & 0.545 & $\begin{array}{c}3 \mathrm{~d}^{8}{ }^{3} \mathrm{~F}_{4}-3 \mathrm{~d}^{7} 4 \mathrm{f} J=5 \\
\left(A=4.2 \cdot 10^{14} s^{-1}\right)\end{array}$ & $\bar{B}$ \\
\hline & & $0.5444(\mathrm{C})$ & 0.545 & $\begin{array}{c}3 \mathrm{~d}^{8}{ }^{3} \mathrm{~F}_{4}-3 \mathrm{~d}^{7} 4 \mathrm{f} J=4 \\
\left(A=1.9 \cdot 10^{14} s^{-1}\right)\end{array}$ & B \\
\hline & & $0.5446(\mathrm{C})$ & 0.545 & $\begin{array}{c}3 \mathrm{~d}^{8}{ }^{3} \mathrm{~F}_{4}-3 \mathrm{~d}^{7} 4 \mathrm{f} J=3 \\
\left(A=2.0 \cdot 10^{14} s^{-1}\right)\end{array}$ & B \\
\hline
\end{tabular}

Table 2. Experimentally observed spectral lines of W ions recommended for diagnostics of W in a fusion plasma. Ionization potentials (I.P.) are taken from [40]. Theoretical wavelengths originate either from GRASP $(\mathrm{G})$ or from the Cowan code $(\mathrm{C})$. Experimental wavelengths are from ASDEX Upgrade. For wavelengths in the SXR (below $2 \mathrm{~nm}$ ) the uncertainty is $\pm 0.001 \mathrm{~nm}$, while for the wavelegths in the VUV range (above $2 \mathrm{~nm}$ ) the uncertainties are $0.005 \mathrm{~nm}$. In the last column problems due to blending with other spectral emissions are indicated. 'B' or ' $\mathrm{BL}$ ' indicate, that the given line is blended with emissions from ionization stages of similar charge or considerably lower charge, respectively. If line blending is considered to cause difficulties for interpreting the spectral line a ' $*$ ' is added.

allowed for a quasi-local measurement. Exploiting this principle, it was possible to determine for the ionization stages Se-like $\mathrm{W}^{40+}$ to $\mathrm{W}^{46+}$ the shape of the fractional abundance vs. electron temperature. The same was possible for the bundle of ionization stages from about Sn-like $\mathrm{W}^{24+}$ to $\mathrm{Y}$-like $\mathrm{W}^{35+}$. These measurements were compared to theoretical predictions using ionization rates, which result from configuration-averaged distorted wave calculations or from the average ion model, and recombination rates from the average ion model. As none of the theoretical predictions could describe the measurements, ad-hoc correction factors, which are independent of electron temperature, were introduced to correct the recombination rates. As an additional boundary condition, the contributions from the different ionization 
Modelling of Measured Tungsten Spectra from ASDEX Upgrade and Predictions for ITER 28

\begin{tabular}{|c|c|c|c|c|}
\hline Ion & I.P. $(\mathrm{eV})$ & $\lambda_{\text {calc }}(\mathrm{nm})$ & transition/configurations & blending \\
\hline $\begin{array}{l}\mathrm{W}^{47+}-\mathrm{W}^{55+} \\
(\mathrm{Co}-\text { like }-\mathrm{K}-\text { like })\end{array}$ & $\begin{array}{l}4180- \\
5348\end{array}$ & $\begin{array}{l}2.7-4.0 \\
0.4-0.7\end{array}$ & $\begin{array}{c}3 \mathrm{p}^{6} 3 \mathrm{~d}^{n}-3 \mathrm{p}^{5} 3 \mathrm{~d}^{n+1} \\
3 \mathrm{p}^{6} 3 \mathrm{~d}^{n}-3 \mathrm{p}^{6} 3 \mathrm{~d}^{n-1} 4 \mathrm{f} \\
\text { and } 3 \mathrm{p}^{6} 3 \mathrm{~d}^{n}-3 \mathrm{p}^{6} 3 \mathrm{~d}^{n-1} 4 \mathrm{p}\end{array}$ & $\begin{array}{l}\mathrm{B}^{*}, \mathrm{BL} \\
\mathrm{B}^{*}, \mathrm{BL}^{*} \\
\mathrm{~B} *, \mathrm{BL}^{*}\end{array}$ \\
\hline $\begin{array}{l}\mathrm{W}^{52+}-\mathrm{W}^{63+} \\
(\mathrm{Ti}-\text { like }-\mathrm{Na}-\text { like })\end{array}$ & $\begin{array}{l}4927- \\
7130\end{array}$ & $1.8-2.4$ & various $\Delta n=0$ transitions & $\mathrm{B}^{*}$ \\
\hline $\begin{array}{l}\mathrm{W}^{57+} \\
(\mathrm{Cl}-\text { like })\end{array}$ & 5671 & $0.13961-0.14003(\mathrm{G})$ & $\begin{array}{c}2 \mathrm{p}^{6} 3 \mathrm{~s}^{2} 3 \mathrm{p}^{52} \mathrm{P}_{3 / 2}-2 \mathrm{p}^{5} 3 \mathrm{~s}^{2} 3 \mathrm{p}^{5} 3 \mathrm{~d} J=\frac{1}{2}, \frac{3}{2}, \frac{5}{2} \\
\left(4 \text { transitions with } A \approx 10^{15} s^{-1}\right)\end{array}$ & \\
\hline $\begin{array}{l}\mathrm{W}^{58+} \\
\text { (S-like) }\end{array}$ & 5803 & $0.13905-0.13924(\mathrm{G})$ & $\begin{array}{c}2 \mathrm{p}^{6} 3 \mathrm{~s}^{2} 3 \mathrm{p}^{4} J=2-2 \mathrm{p}^{5} 3 \mathrm{~s}^{2} 3 \mathrm{p}^{4} 3 \mathrm{~d} J=1,2,3 \\
\left(4 \text { transitions with } A \approx 10^{15} s^{-1}\right)\end{array}$ & \\
\hline $\begin{array}{l}\mathrm{W}^{59+} \\
(\mathrm{P}-\text { like })\end{array}$ & 5936 & $0.13845-0.13894(\mathrm{G})$ & $\begin{array}{c}2 \mathrm{p}^{6} 3 \mathrm{~s}^{2} 3 \mathrm{p}^{3} J=\frac{3}{2}-2 \mathrm{p}^{5} 3 \mathrm{~s}^{2} 3 \mathrm{p}^{3} 3 \mathrm{~d} J=\frac{1}{2}, \frac{3}{2}, \frac{5}{2} \\
\left(4 \text { transitions with } A \approx 10^{15} s^{-1}\right)\end{array}$ & \\
\hline $\begin{array}{l}\mathrm{W}^{60+} \\
(\mathrm{Si}-\text { like })\end{array}$ & 6468 & $0.13810(\mathrm{G})$ & $\begin{aligned} 2 \mathrm{p}^{6} 3 \mathrm{~s}^{2} 3 \mathrm{p}^{2} J & =0-2 \mathrm{p}^{5} 3 \mathrm{~s}^{2} 3 \mathrm{p}^{2} 3 \mathrm{~d} J=1 \\
(A & \left.=2.5 \cdot 10^{15} s^{-1}\right)\end{aligned}$ & \\
\hline $\begin{array}{l}\mathrm{W}^{61+} \\
\text { (Al-like) }\end{array}$ & 6611 & $\begin{array}{l}0.13752(\mathrm{G}) \\
0.13755(\mathrm{G}) \\
7.2687(\mathrm{G})\end{array}$ & $\begin{array}{c}2 \mathrm{p}^{6} 3 \mathrm{~s}^{2} 3 \mathrm{p}^{1} J=\frac{1}{2}-2 \mathrm{p}^{5} 3 \mathrm{~s}^{2} 3 \mathrm{p}^{1} 3 \mathrm{~d} J=\frac{1}{2} \\
\left(A=2.6 \cdot 10^{15} \mathrm{~s}^{-1}\right) \\
2 \mathrm{p}^{6} 3 \mathrm{~s}^{2} 3 \mathrm{p}^{1} J=\frac{1}{2}-2 \mathrm{p}^{5} 3 \mathrm{~s}^{2} 3 \mathrm{p}^{1} 3 \mathrm{~d} J=\frac{3}{2} \\
\left(A=2.6 \cdot 10^{15} s^{-1}\right) \\
2 \mathrm{p}^{6} 3 \mathrm{~s}^{2} 3 \mathrm{p}^{1} J=\frac{1}{2}-2 \mathrm{p}^{6} 3 \mathrm{~s}^{1} 3 \mathrm{p}^{2} J=\frac{1}{2} \\
\left(A=3.5 \cdot 10^{10} s^{-1}\right)\end{array}$ & \\
\hline $\begin{array}{l}\mathrm{W}^{62+} \\
(\mathrm{Mg}-\text { like })\end{array}$ & 6919 & $\begin{array}{r}0.13692(\mathrm{G}) \\
8.0019(\mathrm{G})\end{array}$ & $\begin{array}{c}2 \mathrm{p}^{6} 3 \mathrm{~s}^{2}{ }^{1} \mathrm{~S}_{0}-2 \mathrm{p}^{5} 3 \mathrm{~s}^{2} 3 \mathrm{~d}^{1} \mathrm{P}_{1} \\
2 \mathrm{p}^{6} 3 \mathrm{~s}^{2}{ }^{1} \mathrm{~S}_{0}-2 \mathrm{p}^{6} 3 \mathrm{~s}^{1} 3 \mathrm{p}^{1} J=1 \\
\left(A=1.9 \cdot 10^{10} \mathrm{~s}^{-1}\right)\end{array}$ & \\
\hline $\begin{array}{l}\mathrm{W}^{63+} \\
(\mathrm{Na}-\text { like })\end{array}$ & 7055 & $\begin{array}{l}0.13638(\mathrm{G}) \\
0.13643(\mathrm{G}) \\
7.5977(\mathrm{G})\end{array}$ & $\begin{array}{c}2 \mathrm{p}^{6} 3 \mathrm{~s}^{12} \mathrm{~S}_{1 / 2}-2 \mathrm{p}^{5} 3 \mathrm{~s}^{1} 3 \mathrm{~d} J=\frac{3}{2} \\
\left(A=2.7 \cdot 10^{15} \mathrm{~s}^{-1}\right) \\
2 \mathrm{p}^{6} 3 \mathrm{~s}^{12} \mathrm{~S}_{1 / 2}-2 \mathrm{p}^{5} 3 \mathrm{~s}^{1} 3 \mathrm{~d} J=\frac{1}{2} \\
\left(A=2.6 \cdot 10^{15} s^{-1}\right) \\
2 \mathrm{p}^{6} 3 \mathrm{~s}^{1}{ }^{2} \mathrm{~S}_{1 / 2}-2 \mathrm{p}^{6} 3 \mathrm{p}^{1}{ }^{2} \mathrm{P}_{1 / 2}\end{array}$ & \\
\hline $\begin{array}{l}\mathrm{W}^{64+} \\
(\mathrm{Ne}-\text { like })\end{array}$ & 14760 & $0.13590(\mathrm{G})$ & $2 \mathrm{p}^{6}{ }^{1} \mathrm{~S}_{0}-2 \mathrm{p}^{5} 3 \mathrm{~d}^{1} \mathrm{P}_{1}$ & \\
\hline $\begin{array}{l}\mathrm{W}^{65+} \\
(\mathrm{F}-\text { like })\end{array}$ & 15140 & $0.13341-0.13433(\mathrm{G})$ & $\begin{array}{l}2 \mathrm{p}^{52} \mathrm{P}_{3 / 2}-2 \mathrm{p}^{5} 3 \mathrm{~d} J=\frac{1}{2}, \frac{3}{2}, \frac{5}{2} \\
\left(4 \text { transitions with } A \approx 10^{15} s^{-1}\right)\end{array}$ & \\
\hline $\begin{array}{l}\mathrm{W}^{66+} \\
(\mathrm{O}-\text { like })\end{array}$ & 15520 & $\begin{array}{l}0.13167(\mathrm{G}) \\
0.13190(\mathrm{G})\end{array}$ & $\begin{array}{c}2 \mathrm{p}^{4}{ }^{3} \mathrm{P}_{2}-2 \mathrm{p}^{3} 3 \mathrm{~d} J=3 \\
\left(A=2.2 \cdot 10^{15} s^{-1}\right) \\
2 \mathrm{p}^{4} \mathrm{P}_{2}-2 \mathrm{p}^{3} 3 \mathrm{~d} J=2 \\
\left(A=9.8 \cdot 10^{14} s^{-1}\right)\end{array}$ & \\
\hline $\begin{array}{l}\mathrm{W}^{67+} \\
(\mathrm{N}-\text { like })\end{array}$ & 15900 & $0.12989(\mathrm{G})$ & $\begin{aligned} 2 \mathrm{p}^{3} J & =\frac{3}{2}-2 \mathrm{p}^{2} 3 \mathrm{~d} J=\frac{5}{2} \\
(A & \left.=1.4 \cdot 10^{15} s^{-1}\right)\end{aligned}$ & \\
\hline
\end{tabular}

Table 3. Predicted spectral lines of tungsten ions. Only lines with high intensities are chosen. For the ionization stages of Co-like $\mathrm{W}^{47+}$ to Ar-like $\mathrm{W}^{+56}$ no clear candidates exist, as line blending is leading to quasicontinuous emissions at $2.7-4.0 \mathrm{~nm}$ and $0.4-0.7 \mathrm{~nm}$. Further explanation see table 2 . 
stages to the spectral emissions have been taken into account in an absolute manner. For this purpose, the line intensities from the modelled spectra have been compared to the absolute intensity of the measured line intensities for a flat $\mathrm{W}$ profile. As a result of the correction factors on the recombination rates, also the absolute emissions of the different ionization stages are described more consistently. Looking at the most intense spectral features of $\mathrm{W}$ for the plasmas of ASDEX Upgrade, the focus was placed on the emissions between $0.4-0.8 \mathrm{~nm}$, at $5 \mathrm{~nm}$, between $12-14 \mathrm{~nm}$ and between $10-30 \mathrm{~nm}$. For the emissions at $0.4-0.8 \mathrm{~nm}$, the lines are emitted by ionization stages between $\mathrm{Br}$-like $\mathrm{W}^{39+}$ to about $\mathrm{Mn}$ like $\mathrm{W}^{49+}$. The modelling in that wavelength range agrees well for the rough distribution of emissions vs. the wavelength. The intensities of single spectral lines are predicted typically with deviations of around factor 2, while the wavelengths are predicted within $0.002 \mathrm{~nm}$. For most of the lines, no larger differences occur between the modelling using atomic data from the Cowan code (plane-wave Born approximation) and that using the atomic data from HULLAC or $R$-matrix calculations. For the most intense spectral line in the range, the line at $0.793 \mathrm{~nm}$, larger differences of up to factor 10 are apparent, which are understood to a large exent. For the emissions at $5 \mathrm{~nm}$, the modelling is more difficult, as here hundreds of spectral lines from ionization stages from $\mathrm{Y}$-like $\mathrm{W}^{35+}$ down to possibly I-like $\mathrm{W}^{21+}$ contribute to a spectral feature, which cannot be resolved experimentally. The agreement of modelling is less accurate, as dicrepancies in wavelength predictions occur and certain features like a small local maximum at $6 \mathrm{~nm}$ of the emissions are not accounted for in the modelling. However, the total spectral radiance of the predictions allowing for wavelength mismatch agree with the observed one within a factor of 2 . At higher electron temperatures, additional spectral lines emitted by $\mathrm{Br}$-like $\mathrm{W}^{39+}$ to $\mathrm{Cu}$-like $\mathrm{W}^{45+}$ are observed at the same spectral range, which are also seen in the modelling. The spectral lines at $12-14 \mathrm{~nm}$ emitted by Se-like $\mathrm{W}^{40+}$ to $\mathrm{Cu}$-like $\mathrm{W}^{45+}$ are described to a similar quality, as the spectral lines around $5 \mathrm{~nm}$ being emitted by the corresponding ionization stages. In the spectral range from $10-30 \mathrm{~nm}$, a broad quasicontinuous feature is observed below $\mathrm{T}_{e} \approx 1.3 \mathrm{keV}$. The emitting ionization stages have a lower charge than Pd-like $\mathrm{W}^{28+}$. The atomic data calculations could only be performed down to Sn-like $\mathrm{W}^{24+}$, because of the large number of coupling possibilities for configurations with more than 4 f-electrons, while excited configurations need to be included additionally, exceeded the capabilities of the code. Further emissions around $3 \mathrm{~nm}\left(\mathrm{~T}_{e} \leq 1.1 \mathrm{keV}\right.$ and $\left.\mathrm{T}_{e} \approx 5 \mathrm{keV}\right)$, between $0.6-0.7 \mathrm{~nm}\left(\mathrm{~T}_{e} \approx 0.7 \mathrm{keV}\right)$ and at $8 \mathrm{~nm}\left(\mathrm{~T}_{e} \approx 2 \mathrm{keV}\right)$, which are relatively week, have been experimentally observed, while only the emissions around $3 \mathrm{~nm}$ could be found in the modelling. Finally, the atomic data were used to predict the spectral features of $\mathrm{W}$ in ITER for a standard scenario, in which a central $\mathrm{T}_{e} \approx 18 \mathrm{keV}$ is envisaged. The known emissions will occur at the outer half of the plasma, while new spectral features will be emitted from mid-radius to the plasma center by ionization stages of about Cr-like $\mathrm{W}^{50+}$ to about $\mathrm{C}$-like $\mathrm{W}^{68+}$. Important spectral ranges will be at $0.1-0.15 \mathrm{~nm}$ and $1.8-3.5 \mathrm{~nm}$ for ionization stages of about Co-like $\mathrm{W}^{47+}$ to $\mathrm{C}$-like $\mathrm{W}^{68+}$. Predictions of spectral lines around $8 \mathrm{~nm}$ for the ionization stages above Cl-like $\mathrm{W}^{57+}$ are relatively weak, but seem also an interesting alternative. Further investigations in EBITs and tokamaks are needed to confirm these predictions. 


\section{References}

[1] R. Aymar, P. Barabaschi, and Y. Shimomura, Plasma Physics and Controlled Fusion 44, 519 (2002).

[2] R. Isler, R. Neidigh, and R. Cowan, Phys. Lett. A63, 295 (1977).

[3] E. Hinnov and M. Mattioli, Phys. Lett. A66, 109 (1978).

[4] K. Asmussen et al., Nuclear Fusion 38, 967 (1998).

[5] R. Neu, K. B. Fournier, D. Schlögl, and J. Rice, J. Phys. B: At. Mol. Opt. Phys. 30, 5057 (1997).

[6] R. Neu, K. B. Fournier, D. Bolshukhin, and R. Dux, Physica Scripta T92, 307 (2001).

[7] T. Pütterich, Technical Report No. 10/29, IPP Garching, Germany, doctoral thesis at Universität Augsburg, (2006).

[8] R. Neu, Technical Report No. 10/25, IPP, Garching, Germany (2003).

[9] D. Post et al., At. Data Nucl. Data Tables 20, 397 (1977).

[10] D. Post, J. Abdallah, R. Clark, and N. Putvinskaya, Phys. Plasmas 2, 2328 (1995).

[11] R. Radtke et al., Phys. Rev. A 64, 012720 (2001).

[12] S. B. Utter, P. Beiersdorfer, and E. Träbert, Can. J. Phys. 80, 1503 (2002).

[13] Y. Ralchenko et al., Journal of Physics B: Atomic, Molecular and Optical Physics 40, 3861 (2007).

[14] T. Pütterich et al., J. Phys. B: At. Mol. Opt. Phys. 38, 3071 (2005).

[15] R. D. Cowan, The Theory of Atomic Structure and Spectra (Los Alamos Series in Basic and Applied Sciences) (University of California Press, California, 1981).

[16] R. Neu et al., Plasma Physics and Controlled Fusion 49, B59 (2007).

[17] R. Dux, Technical Report No. 10/30, IPP, Garching, Germany (2006).

[18] K. Behringer, 'Description of the impurity transport code STRAHL', JET-R(87)08, JET Joint Undertaking, Culham (1987).

[19] R. Dux et al., Nuclear Fusion 39, 1509 (1999).

[20] R. Dux, Technical Report No. 10/27, IPP, Garching, Germany (2004).

[21] R. Neu et al., Journal of Nuclear Materials 313-316, 116 (2003).

[22] R. Dux et al., Proc. of the 20th IAEA Conference Fusion Energy (CD-Rom), Vilamoura, Portugal, November 2004 (IAEA, Vienna, 2005), Vol. IAEA-CSP-25/CD, pp. IAEA-CN-116/EX/P6-14.

[23] T. Pütterich et al., to be published

[24] A. G. Peeters, provided the NEOART code

[25] S. P. Hirshman and D. J. Sigmar, Nuclear Fusion 21, 1079 (1981).

[26] W. A. Houlberg, K. C. Shaing, S. P. Hirshman, and M. C. Zarnstorff, Physics of Plasmas 4, 3230 (1997).

[27] A. G. Peeters, Physics of Plasmas 7, 268 (2000).

[28] J. Wesson, Nuclear Fusion 37, 577 (1997).

[29] R. Fonck, A. Ramsey, and R. Yelle, Applied Optics 21, (1982).

[30] E. Unger, Technical Report No. III/187, IPP, Garching, Germany (1992).

[31] D. Bolshukhin et al., Review of Scientific Instruments 72, 4115 (2001).

[32] S. D. Loch et al., Physical Review A (Atomic, Molecular, and Optical Physics) 72, 052716 (2005).

[33] H. P. Summers, The ADAS User Manual, version 2.6 http://adas.phys.strath.ac.uk (2004).

[34] C. P. Ballance and D. C. Griffin, Journal of Physics B: Atomic, Molecular and Optical Physics 39, 3617 (2006).

[35] C. P. Ballance and D. C. Griffin, Journal of Physics B: Atomic, Molecular and Optical Physics 40, 247 (2007).

[36] K. Fournier, At. Data Nucl. Data Tables 68, 1 (1998).

[37] Y. Ralchenko et al., Physical Review A (Atomic, Molecular, and Optical Physics) 74, 042514 (2006).

[38] I. P. Grant, C. T. Johnson, F. A. Parpia, and E. P. Plummer, Comput. Phys. Commun. 55, 425 (1989).

[39] M. Finkenthal et al., Physics Letters A 127, 255 (1988).

[40] A. Kramida and J. Reader, At. Data Nucl. Data Tables 92, 457 (2006).

[41] G. N. Pereverzev, C. Angioni, A. G. Peeters, and O. V. Zolotukhin, Nuclear Fusion 45, 221 (2005).

[42] G. Matthews et al., Physica Scripta T128, 137 (2007). 